\title{
Ammonium glycyrrhizinate-loaded niosomes as a potential nanotherapeutic system for anti-inflammatory activity in murine models
}

This article was published in the following Dove Press journal:

International Journal of Nanomedicine

24 January 2014

Number of times this article has been viewed

\author{
Carlotta Marianecci' \\ Federica Rinaldi' \\ Luisa Di Marzio² \\ Marica Mastriota ${ }^{3}$ \\ Stefano Pieretti ${ }^{3}$ \\ Christian Celia ${ }^{2,4}$ \\ Donatella Paolino ${ }^{5}$ \\ Michelangelo lannone ${ }^{6,7}$ \\ Massimo Fresta $^{5}$ \\ Maria Carafa' \\ 'Department of Drug Chemistry and \\ Technologies, University Sapienza \\ of Rome, Rome, ${ }^{2}$ Department of \\ Pharmacy, University G d'Annunzio of \\ Chieti of Pescara, Chieti, ${ }^{3}$ Department \\ of Therapeutic Research and Medicine \\ Evaluation, Istituto Superiore di \\ Sanità, Rome, Italy; ${ }^{4}$ Department \\ of Nanomedicine, The Methodist \\ Hospital Research Institute, Houston, \\ TX, USA; ${ }^{5}$ Department of Health \\ Sciences, University Magna Graecia \\ of Catanzaro, University Campus \\ $S$ Venuta, Building of BioSciences, \\ Germaneto, ${ }^{6}$ ARPA Calabria, \\ Environmental Epidemiology Center, \\ ${ }^{7} \mathrm{CNR}$, Neuroscience Institute, \\ Pharmacology Section, Complesso \\ "Nini Barbieri", Roccelletta di Borgia, \\ Italy
}

Background: Liquorice extracts demonstrate therapeutic efficacy in treating dermatitis, eczema, and psoriasis when compared with corticosteroids. In this work, nonionic surfactant vesicles (niosomes, NSVs) containing polysorbate 20 (Tween 20), cholesterol, and cholesteryl hemisuccinate at different molar concentrations were used to prepare monoammonium glycyrrhizinate (AG)-loaded NSVs. The anti-inflammatory properties of AG-loaded NSVs were investigated in murine models.

Methods: The physicochemical properties of the NSVs were characterized using dynamic light scattering. The fluidity of the lipid bilayer was evaluated by measuring the fluorescence intensity of diphenylhexatriene. The drug entrapment efficiency of AG was assessed using highperformance liquid chromatography. The physicochemical stability of the NSVs was evaluated as a function of time using dynamic light scattering combined with Turbiscan Lab ${ }^{\circledR}$ Expert analysis. Serum stability was determined by incubating the NSVs with $10 \% \mathrm{v} / \mathrm{v}$ fetal bovine serum. The cytotoxic effects of the NSVs were investigated in human dermal fibroblasts using the Trypan blue dye exclusion assay (for cell mortality) and an MTT assay (for cell viability). Release profiles for the AG-loaded NSVs were studied in vitro using cellulose membranes. NSVs showing the most desirable physicochemical properties were selected to test for in vivo anti-inflammatory activity in murine models. The anti-inflammatory activity of the NSVs was investigated by measuring edema and nociception in mice stimulated with chemical agents.

Results: NSVs showed favorable physicochemical properties for in vitro and in vivo administration. In addition, they demonstrated long-term stability based on Turbiscan Lab Expert analysis. The membrane fluidity of the NSVs was not affected by self-assembling of the surfactants into colloidal structures. Fluorescence anisotropy was found to be independent of the molar ratios of cholesteryl hemisuccinate and/or cholesterol during preparation of the NSVs. The anti-inflammatory AG drug showed no effect on the stability of the NSVs. In vivo experiments demonstrated that AG-loaded NSVs decreased edema and nociceptive responses when compared with AG alone and empty NSVs. In vitro and in vivo results demonstrated that $\mathrm{pH}$ sensitive and neutral NSVs show no statistical significant difference.

Conclusion: NSVs were nontoxic and showed features favorable for potential administration in vivo. In addition, neutral NSVs showed signs of increased anti-inflammatory and antinociceptive responses when compared with AG.

Keywords: niosomes, ammonium glycyrrhizinate, $\mathrm{pH}$ sensitivity, cytotoxicity, inflammation

\section{Introduction}

A major focus in pharmaceutical science is the use of surfactants as biomaterials to prepare nanotherapeutics, particularly niosomes. ${ }^{1-5}$ Recently, niosomes have been proposed as an alternative to liposomes for drug delivery and potentially could be used as nanocarriers in theranostics. ${ }^{6,7}$
Correspondence: Maria Carafa Department of Drug Chemistry and Technologies, Sapienza Università $\mathrm{di}$ Roma, P le A Moro 5, Rome 00185, Italy Tel +390649913603 Fax +390649913133

Email maria.carafa@uniromal.it 
Although niosomes have shown promise in pharmaceutical science and nanotherapy, smart nonionic surfactant vesicles (NSVs) have been developed to further improve their potential as nanomedicines. In addition, $\mathrm{pH}$-sensitive niosomes have been designed to respond to biological stimuli and be used for diagnosis and therapy. ${ }^{8,9}$

Modification of $\mathrm{pH}$ plays a pivotal role in a variety of diseases. For example, tumor and inflammatory tissues often show decreased $\mathrm{pH}$ levels, increased interstitial pressure, and activation of inflammatory mediators when compared with healthy tissues. ${ }^{10,11}$ Therefore, the physiological conditions of tumors and inflammatory tissues can be used strategically to selectively target nanotherapeutics with increased affinity for an acidic $\mathrm{pH}$ microenvironment (ie, 5.8-7.2) compared with the neutral $\mathrm{pH}$ of healthy tissues (ie, 7.0-7.4). ${ }^{12-15}$

Importantly, an acidic $\mathrm{pH}$ has also been found inside membrane-bound organelles (eg, endosomes and lysosomes) and generally enables metabolic degradation of nonself compounds and microorganisms. ${ }^{16-18} \mathrm{An}$ acidic $\mathrm{pH}$ can also modulate the release kinetics of drugs and genetic materials loaded into $\mathrm{pH}$-sensitive nanotherapeutics and provide a novel approach to prepare $\mathrm{pH}$-responsive nonionic surfactant nanotherapeutics for treating inflammatory diseases. ${ }^{19,20}$ Monoammonium glycyrrhizinate (AG), a natural compound obtained from Glycyrrhiza glabra, was found to show antiinflammatory activity in vitro and in vivo, in addition to being used in humans as a model drug to investigate the effect of niosomes in animal models of induced edema and pain..$^{21-23}$

In this work, we investigated the use of NSVs as a potential nanotherapeutic agent for anti-inflammatory treatment in vivo. The niosomes were prepared using Tween 20 and cholesterol as previously reported. ${ }^{24}$ Cholesteryl hemisuccinate (CHEMS) was added to the NSVs, thereby obtaining $\mathrm{pH}$-sensitive vesicles. CHEMS can destabilize the NSV bilayer at acidic $\mathrm{pH}$, thus promoting the release of a payload. Furthermore, CHEMS improves the stability of NSVs. ${ }^{25}$

This potential nanotherapeutic strategy was characterized by dynamic light scattering (ie, average size, size distribution, and zeta potential), fluorescent anisotropy, drug entrapment efficiency, release kinetics, and plasma stability.

The in vitro and in vivo efficacy of the AG-loaded NSVs was evaluated further using cellular and animal models, respectively. The results demonstrated that empty NSVs are nontoxic and have promising features for potential administration in vivo. AG-loaded NSVs also achieved an improved anti-inflammatory and antinociceptive response when compared with the free drug.

\section{Materials and methods Materials}

Tween 20, cholesterol, CHEMS, Sephadex G75, HEPES salt (N-(2-idroxyethyl) piperazine-N-(2-ethanesulfonic acid)), zymosan A, formalin, 3-(4,5-dimethylthiazol-2yl)-2,5-diphenyltetrazolium bromide (MTT) salt, and Trypan blue were purchased from Sigma-Aldrich SRL (Milan, Italy). Fetal bovine serum, $0.1 \%$ trypsin $/ 0.02 \%$ ethylenediamine tetraacetic acid, phosphate-buffered saline, and Dulbecco's Modified Eagle's Medium were purchased from Gibco ${ }^{\circledR}$ Cell Culture Basics (Life Technology Italia, Monza, Italy). Diphenylhexatriene was obtained from Acros Organics BVBA (Geel, Belgium). The AG was a kind gift from Bernett (Pavia, Italy). The cellulose membrane dialysis tubing was purchased from Spectrum Laboratories (Prodotti Gianni SpA, Milan, Italy). Cellulose filters $(0.45 \mu \mathrm{m})$ were obtained from Millipore (Billerica, MA, USA). Tissue culture test plates were purchased from TPP Tissue Cell Culture Products (Delchimica Scientific Glassware, Naples, Italy). Male CD-1 mice were purchased from Harlan Laboratories (Correzzana, Italy). All other chemicals used during the experiments were of analytical grade and no additional purification was carried out before analysis. Double-distilled water was used throughout the study.

\section{Vesicle preparation and purification}

The thin-layer evaporation method was used to prepare $\mathrm{NSVs}^{26}$ containing Tween 20, cholesterol, and CHEMS at different molar ratios (Table 1). The organic solvent was removed under vacuum and the obtained film was hydrated with $5 \mathrm{~mL}$ of HEPES buffer (10 mM, pH 7.4). Lipid suspension was vortex-mixed for 5 minutes at room temperature followed by a 5-minute sonication at $60^{\circ} \mathrm{C}$ using a tapered microtip operating at $20 \mathrm{kHz}$ and an amplitude of $16 \%$ (VibraCell-VCX 400 , Sonics, Taunton, MA, USA). When required, AG $(0.5 \%$, $0.75 \%, 1 \% \mathrm{w} / \mathrm{v})$ was added to HEPES buffer $(10 \mathrm{mM}, \mathrm{pH} 7.4)$ before hydrating the lipid film. Diphenylhexatriene (final concentration $200 \mu \mathrm{M}$ ) was co-dissolved into the surfactant/ cholesterol mixture to obtain fluorescent NSVs. Empty NSVs

Table I Surfactants and lipids used to obtain nonionic surfactant vesicles

\begin{tabular}{llll}
\hline Samples & Tween 20 & Cholesterol & $\begin{array}{l}\text { Cholesteryl } \\
\text { hemisuccinate }\end{array}$ \\
\hline FI & $15.00 \mathrm{mM}$ & $15.00 \mathrm{mM}$ & - \\
F2 & $15.00 \mathrm{mM}$ & $11.25 \mathrm{mM}$ & - \\
F3 & $15.00 \mathrm{mM}$ & $2.00 \mathrm{mM}$ & $13 \mathrm{mM}$ \\
\hline
\end{tabular}


were prepared using the same procedure and used as a control during the experiments.

NSVs were purified using gel permeation chromatography (glass column $50 \times 1.2 \mathrm{~cm}$ ) in Sephadex G75 (stationary phase) using HEPES buffer (10 mM, pH 7.4, mobile phase). Purification of the NSVs was carried out at room temperature; $5 \mathrm{~mL}$ of NSVs were eluted through the column and $20 \mathrm{~mL}$ of the purified formulations were finally collected.

\section{Dynamic light scattering analysis}

The average size and size distribution (polydispersity index) of the NSVs were characterized using dynamic light scattering. NSVs were diluted 1:100 v/v using HEPES buffer (10 mM, pH 7.4) to avoid multiscattering. Buffer solution was previously filtered through $0.45 \mu \mathrm{m}$ cellulose filters (Millex ${ }^{\circledR}$ Syringe Filters, Millipore) to eliminate dust particles. The average size of the NSVs and their size distribution were measured at $25^{\circ} \mathrm{C}$ using a Nano ZS90 (Malvern Instruments Ltd, Malvern, UK) at a scattering angle of $90.0^{\circ}$. The following parameters were set up during the analysis: medium refractive index 1.330 , medium viscosity $1.0 \mathrm{mPa} \cdot \mathrm{s}$, and a dielectric constant of 80.4. A third-order cumulant fitting autocorrelation function was used to calculate mean size and polydispersity index.

Dynamic light scattering was further used to evaluate the zeta potential. For this, NSVs were appropriately diluted $(1: 10 \mathrm{v} / \mathrm{v})$ in filtered HEPES $(10 \mathrm{mM}, \mathrm{pH} 7.4)$ at $25^{\circ} \mathrm{C}$. Laser Doppler anemometry was used during analysis. The zeta potential value was measured from the electrophoresis mobility using a Smoluchowski constant $\mathrm{F}\left(\mathrm{K}_{\mathrm{a}}\right)$ of 1.5.

\section{Freeze-fracture transmission electron microscopy}

The NSVs were further characterized using freeze-fracture transmission electron microscopy. Samples were vitrified using 30\% v/v glycerol solution and then frozen in Freon 22. Solid samples were freeze-fractured $\left(-105^{\circ} \mathrm{C}, 10^{-6} \mathrm{mmHg}\right)$ in a freeze-fracture holder and evaporated under a platinum/ carbon gun. Samples were washed three times in deionized distilled water and dropped onto Formvar-coated grids before analysis. The analysis was carried out using a CM10 Transmission Electron Microscope (Philips, Eindhoven, the Netherlands) operating at $100 \mathrm{kV}$.

\section{Physicochemical stability using Turbiscan ${ }^{\circledR}$ Lab Expert}

The long-term stability of the NSVs was performed using Turbiscan Lab Expert (Formulaction, L'Union, France) as previously reported. ${ }^{27}$ Briefly, samples were scanned until $10 \mathrm{~mm}$ in height and detection was performed every minute for one hour. Analysis was performed as a function of back scattering (BS) of the NSVs (Supplementary material).

\section{Physicochemical stability in serum}

Physicochemical stability studies of the AG-loaded NSVs were carried out by evaluating their average size, polydispersity index, and zeta potential when stored at $4{ }^{\circ} \mathrm{C}$ and $25^{\circ} \mathrm{C}$ for 90 days. Samples were analyzed at different time points (1,30, 60, and 90 days) and their average size and zeta potential were measured as previously reported.

The serum stability of the NSVs was further evaluated by coincubating AG-NSVs $(250 \mu \mathrm{L})$ diluted in $2.25 \mathrm{~mL}$ of HEPES buffer (10 mM, pH 7.4) supplemented with $(10 \% \mathrm{v} / \mathrm{v})$ and without $(0 \% \mathrm{v} / \mathrm{v})$ fetal bovine serum. The percentage of AG leaked from the NSVs was measured 3 hours and 24 hours after incubation by dissolving the NSVs with isopropyl alcohol. The concentration of AG loaded into the NSVs was measured using high-performance liquid chromatography (HPLC) as previously reported (Supplementary material). ${ }^{23}$

\section{Fluidity analysis}

Diphenylhexatriene $(200 \mu \mathrm{M})$ was used to prepare fluorescently labeled NSVs, which were stabilized for 3 hours at room temperature before the experiments. The fluidity of the NSVs was assessed by measuring the fluorescence anisotropy of diphenylhexatriene, as previously reported. ${ }^{27}$

\section{Drug entrapment efficiency and in vitro release studies}

AG-loaded NSVs were evaluated using HPLC as previously reported. Purified NSVs were disrupted using isopropyl alcohol (in a ratio of $1: 1 \mathrm{v} / \mathrm{v}$ ) and entrapment efficiency was quantified as reported in the Supplementary material.

The in vitro release experiments were carried out using dialysis tubes (molecular weight cutoff 8,000 and $5.5 \mathrm{~cm}^{2}$ diffusing area) at $37^{\circ} \mathrm{C}$ in HEPES buffer (10 mM, pH 7.4). Free AG was used as the control. The AG concentration was measured using HPLC at different time points over 1-10 hours. The release profile of AG was evaluated as reported in the Supplementary material. The permeability coefficient of the niosomal bilayer $\left(\mathrm{P}_{\mathrm{bl}}\right)$ was evaluated using a linear expression developed by $\mathrm{Ho}$ et al as previously reported (Supplementary material). ${ }^{28}$ 


\section{In vitro cytotoxicity experiments}

Primary human dermal fibroblast cell culture and cytotoxicity of NSVs

Primary human dermal fibroblasts were isolated from the skin of consenting patients who had undergone abdominal reduction surgery. ${ }^{29}$ Briefly, skin slides pretreated with type IA collagenase and type IVS hyaluronidase at $37^{\circ} \mathrm{C}$ for 45 minutes were washed and seeded into tissue culture plates and further incubated using high-glucose Dulbecco's Modified Eagle's Medium. ${ }^{30}$ The cell culture medium and skin slides were removed and primary human dermal fibroblasts were then incubated with fresh complete Dulbecco's Modified Eagle's Medium until at $\sim 70 \%$ confluence. The cytotoxicity of the empty NSVs in primary human dermal fibroblasts was evaluated using Trypan blue dye exclusion (cell mortality) and MTT (cell viability) assays. Details about the in vitro procedures and the cytotoxicity of the NSVs are reported in the Supplementary material.

\section{In vivo evaluation of anti-inflammatory and antinociceptive activity}

The anti-inflammatory and antinociceptive activity of AG was evaluated in vivo using male CD-1 mice weighing 25-30 g. The animals were fed on a standard chow pellet diet with free access to water, maintained on 12-hour light/dark cycles under a controlled temperature $\left(22^{\circ} \mathrm{C} \pm 1^{\circ} \mathrm{C}\right)$, and housed for at least one week before the experiments in colony cages (seven mice per cage). The research protocol was approved by the Service for Biotechnology and Animal Welfare of the Istituto Superiore di Sanità and authorized by the Italian Ministry of Health, according to Legislative Decree 116/92, which implements the European Directive 86/609/EEC on laboratory animal protection in Italy. Animal welfare was routinely checked and complied with the ethical laws established by the Service for Biotechnology and Animal Welfare. Formulation 1 (F1AG) and formulation 3 (F3AG) containing AG $1 \% \mathrm{w} / \mathrm{v}$ and providing the best physicochemical features were selected for the in vivo experiments. F1 AG and F2AG showed comparable physicochemical properties, and for this reason only F1AG, characterized in depth in our previous studies, was selected for in vivo comparison with the $\mathrm{pH}$ sensitive niosomes. ${ }^{24-26,31}$ Empty F1 and F3 formulations were used as controls during the in vivo experiments.

\section{Edema induced by zymosan}

Edema was induced by subcutaneous injection of $2.5 \% \mathrm{w} / \mathrm{v}$ zymosan A dissolved into saline solution $(\mathrm{NaCl} 0.9 \% \mathrm{w} / \mathrm{v})$ into the dorsal surface of the right hind paw ( $20 \mu \mathrm{L}$ per paw).
Paw volume was measured three times before and at different time points (1, 2, 3, 4, 24, 48, and 72 hours) after the injections, and edema was measured using a hydroplethysmometer to monitor paw volume modification. An increase in paw volume was used to demonstrate the development of edema in treated animals (Ugo Basile, Comerio, Italy, unpublished data). F1, F1AG, F3, F3AG, and free AG (1\% w/v) in solution were subcutaneously administered to the dorsal surface of the mouse paw ( $40 \mu \mathrm{L}$ per paw) 30 or 120 minutes before injection of zymosan A. The increase in paw volume was measured as the difference in percentage between the paw volume at different time points and the basal paw volume. ${ }^{33}$

\section{Nociception induced by formalin}

Subcutaneous injection of formalin solution $(1 \% \mathrm{w} / \mathrm{v})$ into the hind paw $(20 \mu \mathrm{L}$ per paw) induces nociceptive responses (eg, licking and/or biting the injected paw) in mice. Both behaviors indicate the degree of pain experienced by the animals. Before this test, the mice were placed in a Plexiglas observation cage $(30 \times 14 \times 12 \mathrm{~cm})$ one hour before administration of formalin to allow acclimation, and after administration of formalin, the total time (seconds) that each animal spent licking or biting its paw was recorded during the early and late phase of formalin-induced nociception. ${ }^{34} \mathrm{~F} 1, \mathrm{~F} 1 \mathrm{AG}, \mathrm{F} 3$, F3AG, and free AG 1\% w/v solution were subcutaneously injected into the dorsal surface of the mouse paw $(40 \mu \mathrm{L}$ per paw) 30 or 120 minutes before administration of formalin.

\section{Statistical analysis}

The data are expressed as the average \pm standard error of the mean of three different experiments. The statistical significance of data from the in vitro studies was tested using the $t$-test; two-way analysis of variance testing followed by Bonferroni's post hoc comparisons was used to evaluate the statistical significance of data from the edema experiments, while one-way analysis of variance followed by Dunnett's multiple comparison test was used to evaluate the statistical significance of data from both the early phase and the late phase of the formalin test. Data has been considered statistically significant for $P<0.05\left({ }^{*} P<0.05 ;{ }^{*} P<0.01\right.$; $* * * P<0.001)$.

\section{Results}

\section{Characterization and physical stability of NSVs}

The thin-layer evaporation method was used to prepare NSVs ${ }^{29,34}$ containing different molar ratios of Tween 20, cholesterol, and CHEMS, as shown in Table 1. 
Surfactant, cholesterol, and cholesterol derivatives were self-assembled to obtain $\mathrm{pH}$-sensitive NSVs. ${ }^{8,35-39}$ The different molar ratio of compounds herein reported improves the stability of NSVs and provides suitable nanocarriers for in vitro and in vivo administration. Freeze-fracture microscopy analysis demonstrated that presence of the drug does not modify the morphology or supramolecular structure of NSVs (Figure 1). These results are in agreement with those obtained by dynamic light scattering analysis and demonstrate that NSVs have a suitable size and shape for potential administration in vitro and in vivo. The physicochemical features of NSVs were modified by loading of AG inside the aqueous compartment (Table 2). The average size and polydispersity index were increased by entrapping AG, while the zeta potential values were not affected by the drug (Table 2 ). Turbiscan Lab Expert analysis demonstrated the long-term stability of NSVs (Figure S1). The positive or negative profile of $\triangle \mathrm{BS}$ was below $2 \%$ compared with the reference threshold and overlapped the baseline during the full-scale range of detection (Figure 2). No significant variation in $\triangle \mathrm{BS}$ occurred during analysis of NSVs prepared using Tween 20 and cholesterol or Tween 20, cholesterol, and CHEMS (Figure 2). The $\Delta \mathrm{BS}$ overlapped in both formulations during analysis (Figure 2). Additionally, the entrapment of AG inside NSVs did not modify the stability of the colloidal nanotherapeutics (Figure 2). Assembling of CHEMS and/or cholesterol into the NSVs does not alter their anisotropy.

The results demonstrate that the fluidity of the NSV bilayer is independent of the molar ratio of cholesterol and CHEMS and also independent of inclusion of AG inside these vesicular structures (Table 2). There was no variation
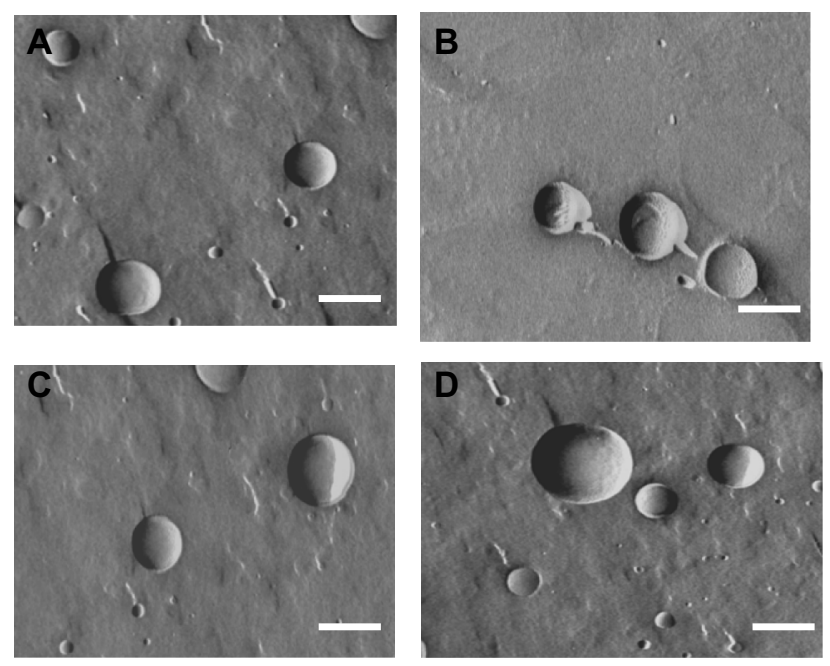

Figure I Transmission electron micrographs of nonionic surfactant vesicles after freeze-fracture. FI (A), FIAG (B), F3 (C), and F3AG (D). Scale bars, I50 nm. in anisotropy values between the empty and loaded vesicles. This result could be explained by selective distribution of AG (a hydrophilic drug) in the aqueous core. The entrapment efficiency of the NSVs was greater than $9 \%$. The increase in entrapment efficiency obtained by increasing the percentage of AG in the NSVs from $0.5 \%$ to $1 \% \mathrm{w} / \mathrm{v}$ was not considered to be statistically significant $(10.0 \%, 9.1 \%$, and $9.7 \%$ for formulations 1, 2, and 3, respectively, Table 2). A further increase in $\mathrm{AG}$ over $1 \% \mathrm{w} / \mathrm{v}$ did not achieve any significant improvement in entrapment efficiency, thus not providing narrow size distribution of vesicles (data not shown). Based on these considerations, formulations containing AG 1\% $\mathrm{w} / \mathrm{v}$ were selected for further characterization in vitro and in vivo.

Both empty and AG-loaded NSVs were stable for at least 90 days in HEPES buffer (10 mM, pH 7.4) at $4^{\circ} \mathrm{C}$ (Figure 3 ). Average size, polydispersity index, and zeta potential showed no statistically significant changes after 90 days (Figure 3). In contrast, NSVs with and without AG were unstable when stored at $25^{\circ} \mathrm{C}$ (Figure 4).

Composition of the NSVs did not affect the fluidity of the bilayer (Table 2). Fluorescence anisotropy demonstrated that the NSVs containing cholesterol or cholesterol and CHEMS at different molar ratios were similar and without any statistically significant differences (Table 2). These data are in agreement with the kinetics of release of AG from the NSVs, which demonstrated a biphasic profile in vitro (Figure 5). This effect depends on the AG permeability coefficient through the bilayer and is not related to modification of the structure of the NSVs (Table 2). A lag time was observed in the first 3 hours, followed by zero-order kinetics until 8 hours of incubation.

Serum stability was further investigated to determine the effect of the in vivo environment on NSVs. Samples were incubated in HEPES buffer (10 mM, pH 7.4) with (10\% v/v) or without $(0 \% \mathrm{v} / \mathrm{v})$ fetal bovine serum. The results show that serum content does not modify the profile of AG release from NSVs at pH 7.4 (less than 0.05 of the entrapped drug) after 3 hours (Table S1).

\section{In vivo evaluation of anti-inflammatory and antinociceptive activity}

Our in vivo results are reported in Figure 6. Figure 6A shows the effect of NSVs on the edema induced by zymosan, and Figure 6B shows the effects of NSVs on the nociception induced by formalin (Figure 6A). The preliminary data demonstrate that empty NSVs (Table 1) have an effect similar to that of HEPES buffer (10 mM, pH 7.4, control) 
Table 2 Physicochemical characterization of nonionic surfactant vesicles

\begin{tabular}{lllllll}
\hline Samples & $\begin{array}{l}\text { Average } \\
\text { size }(\mathbf{n m})\end{array}$ & $\begin{array}{l}\text { Zeta } \\
\text { potential }(\mathbf{m V})\end{array}$ & PDI & $\begin{array}{l}\text { Fluorescence } \\
\text { anisotropy }\end{array}$ & EE (\%) & $\begin{array}{l}\text { Coefficient of } \\
\text { permeability }(\mathbf{c m} / \mathbf{h o u r})\end{array}$ \\
\hline FI & $140.8 \pm 1.6$ & $-30.4 \pm 1.0$ & $0.22 \pm 0.04$ & 0.20 & - & - \\
FIAG & $198.7 \pm 5.7$ & $-28.2 \pm 1.1$ & $0.41 \pm 0.01$ & 0.24 & $10.01 \pm 1.2$ & $1.13 .10^{-5} \pm 4.10^{-7}$ \\
F2 & $133.1 \pm 1.8$ & $-28.5 \pm 0.1$ & $0.18 \pm 0.02$ & 0.21 & - & - \\
F2AG & $189.2 \pm 1.2$ & $-27.8 \pm 0.6$ & $0.25 \pm 0.01$ & 0.21 & $9.1 \pm 1.1$ & $1.07 .10^{-5} \pm 2.10^{-7}$ \\
F3 & $192.8 \pm 2.5$ & $-40.6 \pm 0.7$ & $0.30 \pm 0.01$ & 0.21 & - & - \\
F3AG & $217.4 \pm 3.5$ & $-39.7 \pm 1.5$ & $0.24 \pm 0.03$ & 0.25 & $9.7 \pm 1.5$ & $1.15 .10^{-5} \pm 5.10^{-7}$ \\
\hline
\end{tabular}

Note: The data represent the average \pm standard error of the mean of different experiments.

Abbreviations: AG, monoammonium glycyrrhizinate solution ( $\% \mathrm{w} / \mathrm{v})$; PDI, polydispersity index; EE, entrapment efficacy.

on both edema and nociception (data not reported). For this reason, only the in vivo results for control animals treated with HEPES buffer (10 mM, pH 7.4) are reported (Figure 6). In the controls, subcutaneous injection of $2.5 \%$ zymosan into the dorsal surface of the right hind paw $(20 \mu \mathrm{L}$ per paw) resulted in an increase in paw volume that reached a maximal value 3-4 hours after injection. This increase was followed by a reduction in the next $24-48$ hours (Figure 6A). Local injection of free AG $1 \% \mathrm{w} / \mathrm{v}$ solution 2 hours before administration of zymosan resulted in a slight but not statistically significant decrease in paw edema, as shown 1-2 hours after administration of zymosan (Figure 6A). AG-loaded NSVs (F1AG), prepared using Tween 20 and cholesterol at a molar ratio of 1:1 (sample 1), increased antiinflammatory activity in the animal model. In fact, the effect

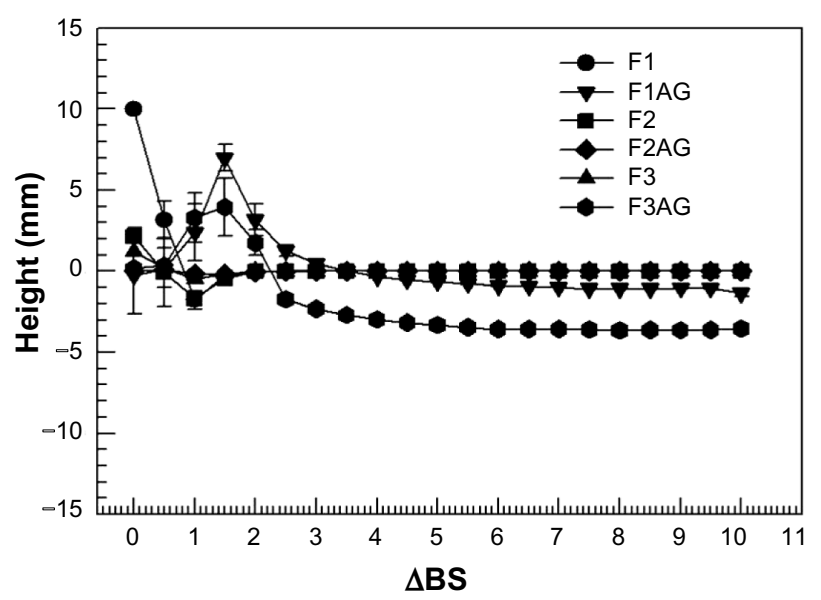

Figure 2 Niosomes on Turbiscan Lab ${ }^{\circledR}$ Expert. The $\Delta \mathrm{BS}$ profile of nonionic surfactant vesicles was exported using Turbiscan EasySoft Converter. The data were previously collected using Turbiscan Lab ${ }^{\circledR}$ Expert software (Formulaction, L'Union, France). Samples were evaluated as a function of time (0-I hour) and height of the samples $(0-10 \mathrm{~mm})$. Samples $5 \mathrm{~mL}$ in volume were run during the analysis. The Turbiscan EasySoft Converter allowed exporting and listing of the raw data into an Excel file. The exported data was then processed and the average \pm standard error of the mean was calculated and represented graphically. The data shown are the average \pm standard error of the mean of three different experiments.

Abbreviations: AG, monoammonium glycyrrhizinate solution ( $1 \% \mathrm{w} / \mathrm{v})$; $\mathrm{BS}$, back scattering. of $\mathrm{AG} 1 \% \mathrm{w} / \mathrm{v}$ was potentiated and edema was decreased in animals treated with F1AG (Table 1) at 1-4 hours after administration of zymosan (Figure 6A). The reduction in paw edema obtained by administration of F1AG was still present 24 and 48 hours after administering zymosan, but was not significantly different from that in control animals given HEPES buffer or empty F1 vesicles. There was a dramatic decrease in development of edema in animals treated with F3AG (Figure 6A). In fact, the antiedema effect appeared after one hour and was sustained until 48 hours after administration of zymosan (Figure 6A). However, this effect was not statistically significant compared with animals treated using HEPES buffer or empty F3 vesicles for the same incubation period. The same effects were obtained using AG 1\% w/v solution and formulations F1AG and F3AG in the formalin test (Figure 6B). After administration of formalin, the nociceptive response shows a biphasic trend, consisting of an early phase occurring $0-10$ minutes after injection of formalin due to direct stimulation of peripheral nociceptors, followed by a late prolonged phase occurring at 20-40 minutes, reflecting the response to inflammatory pain. In the early phase of the formalin test, none of the samples administered was able to modify the effects induced by the aldehyde solution. A slight decrease in nociceptive stimuli was obtained in the early phase after administration of free AG and F1AG, but this effect was not statistically significant when compared with that in animals treated using HEPES buffer or empty F1vesicles (Figure 6B). In contrast, a significant reduction in nociceptive stimuli was observed after administration of F1AG in the late phase of the formalin test (Figure 6B). Administration of free AG did not modify the response to formalin, as demonstrated in the late phase of the formalin test (Figure 6B). F3AG was able to reduce the licking behavior induced by formalin in the late phase of the test, but the reduction was not significant compared with that in animals treated using HEPES buffer or empty F3 vesicles (Figure 6B). 


\begin{tabular}{|l|c|l|l|l|}
\hline Samples & $\begin{array}{c}\zeta \text { potential }(\mathrm{mV}) \\
\mathrm{t}=1 \text { day }\end{array}$ & $\begin{array}{c}\zeta \text { potential }(\mathrm{mV}) \\
\mathrm{t}=90 \text { days }\end{array}$ & PDI $\mathrm{t}=1$ day & $\begin{array}{c}\text { PDI } \\
\mathrm{t}=90 \text { days }\end{array}$ \\
\hline $\mathrm{F} 1$ & $-30 \pm 3$ & $-36 \pm 2$ & $0.15 \pm 0.03$ & $0.21 \pm 0.05$ \\
\hline F1AG & $-26 \pm 5$ & $-31 \pm 3$ & $0.19 \pm 0.04$ & $0.27 \pm 0.04$ \\
\hline F2 & $-28 \pm 3$ & $-30 \pm 2$ & $0.22 \pm 0.01$ & $0.27 \pm 0.03$ \\
\hline F2AG & $-23 \pm 4$ & $-28 \pm 3$ & $0.25 \pm 0.02$ & $0.27 \pm 0.02$ \\
\hline F3 & $-40 \pm 2$ & $-39 \pm 3$ & $0.15 \pm 0.01$ & $0.22 \pm 0.03$ \\
\hline F3AG & $-39 \pm 3$ & $-36 \pm 2$ & $0.21 \pm 0.03$ & $0.25 \pm 0.04$ \\
\hline
\end{tabular}

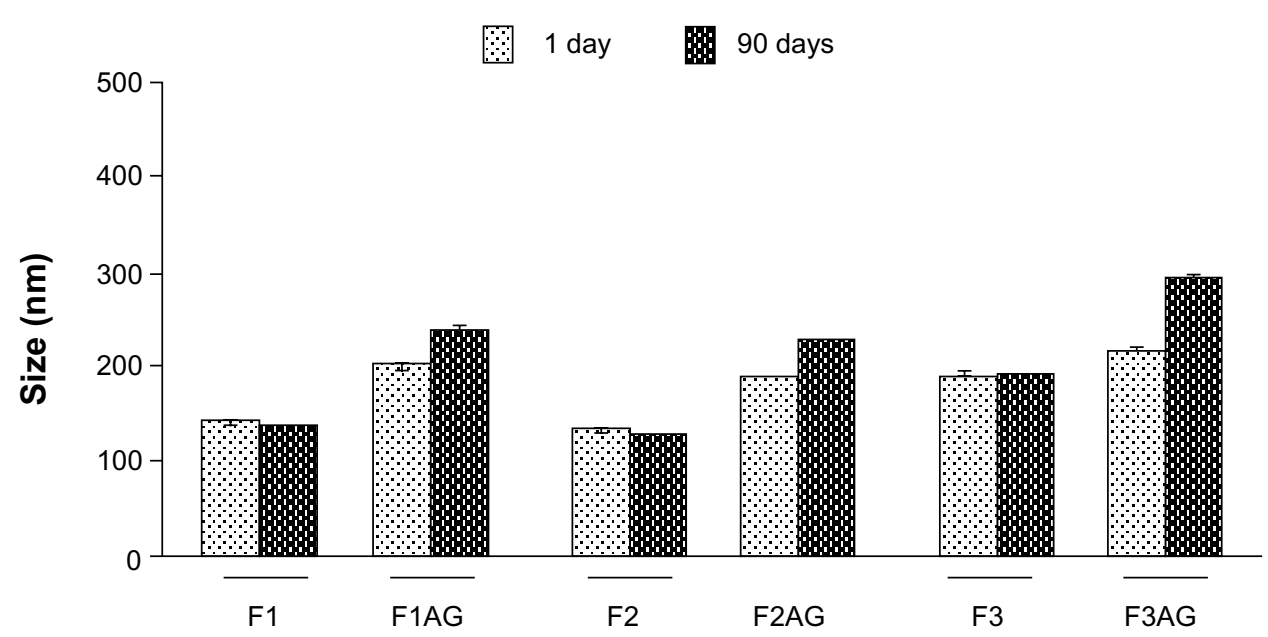

Figure 3 Physical stability of nonionic surfactant vesicles at $4^{\circ} \mathrm{C}$. Their average size and zeta potential were evaluated by dynamic light scattering at defined time intervals (days) at $4^{\circ} \mathrm{C}$. The data shown are the average \pm standard error of the mean of three experiments.

Abbreviations: PDI, polydispersity index; AG, monoammonium glycyrrhizinate solution (I\% w/v).

\section{Evaluation of cytotoxicity}

The F1 formulation showing the best physicochemical parameters and in vivo nociceptive response was selected for in vitro experiments in primary human dermal fibroblasts. These in vitro experiments demonstrated that neither empty nor AG-loaded NSVs had any significant cytotoxic effects up to a concentration of $1 \mu \mathrm{M}$ (Figure 7A). At least $90 \%$ of cell viability was carried out from 0.001 to the $1 \mu \mathrm{M}$ concentration of the formulation at different incubation times. No significant differences in cell viability were obtained when comparing the empty (Figure 7B) and AG-loaded NSVs (Figure 7A). Primary human dermal fibroblast cell viability decreased to less than $80 \%$ when treated with NSVs at a concentration of $10 \mu \mathrm{M}$. Cell viability shows a time-dependent and dose-dependent effect and cytotoxicity occurred after 48 hours of incubation (Figure 7A and B). Significant cytotoxicity was only obtained by incubating primary human dermal fibroblasts with a high NSV concentration for 24 hours (Figure 7A and B).
The Trypan blue dye exclusion assay showed results similar to those of the MTT assay. Cell death percentages, obtained for primary human dermal fibroblasts and evaluated at different incubation times (24, 48, and 72 hours), were comparable with the cell viability data (not reported).

\section{Discussion}

Glycyrrhizinic acid and its derivatives have been approved for use in the US since 1985 as an additive in foods and obtained the Generally Recognized as Safe (GRAS) status in the same year. ${ }^{40}$ Nevertheless, glycyrrhizinic acid and AG are actually registered as flavoring substances according to the guidelines for foods adopted by Commission Decision 1999/217/EC, with specific limits fixed concerning their addition to foods, cosmetic products, and pharmaceutical formulations. ${ }^{41,42}$

In this study, we investigated the in vivo anti-inflammatory activity of AG to evaluate its potential therapeutic efficacy in 


\begin{tabular}{|l|c|c|c|c|}
\hline \multicolumn{1}{|c|}{ Samples } & $\begin{array}{c}\zeta \text { potential }(\mathrm{mV}) \\
\mathrm{t}=1 \text { day }\end{array}$ & $\begin{array}{c}\zeta \text { potential }(\mathrm{mV}) \\
\mathrm{t}=90 \text { days }\end{array}$ & PDI $\mathrm{t}=1$ day & $\begin{array}{c}\text { PDI } \\
\mathrm{t}=90 \text { days }\end{array}$ \\
\hline F1 & $-30 \pm 3$ & $-23 \pm 2$ & $0.15 \pm 0.03$ & $0.22 \pm 0.03$ \\
\hline F1AG & $-26 \pm 5$ & $-21 \pm 3$ & $0.19 \pm 0.04$ & $0.35 \pm 0.01$ \\
\hline F2 & $-28 \pm 3$ & $-29 \pm 2$ & $0.22 \pm 0.01$ & $0.25 \pm 0.02$ \\
\hline F2AG & $-23 \pm 4$ & $-11 \pm 3$ & $0.25 \pm 0.02$ & $0.42 \pm 0.05$ \\
\hline F3 & $-40 \pm 2$ & $-40 \pm 3$ & $0.15 \pm 0.01$ & $0.23 \pm 0.01$ \\
\hline F3AG & $-39 \pm 3$ & $-20 \pm 2$ & $0.21 \pm 0.03$ & $0.45 \pm 0.06$ \\
\hline
\end{tabular}

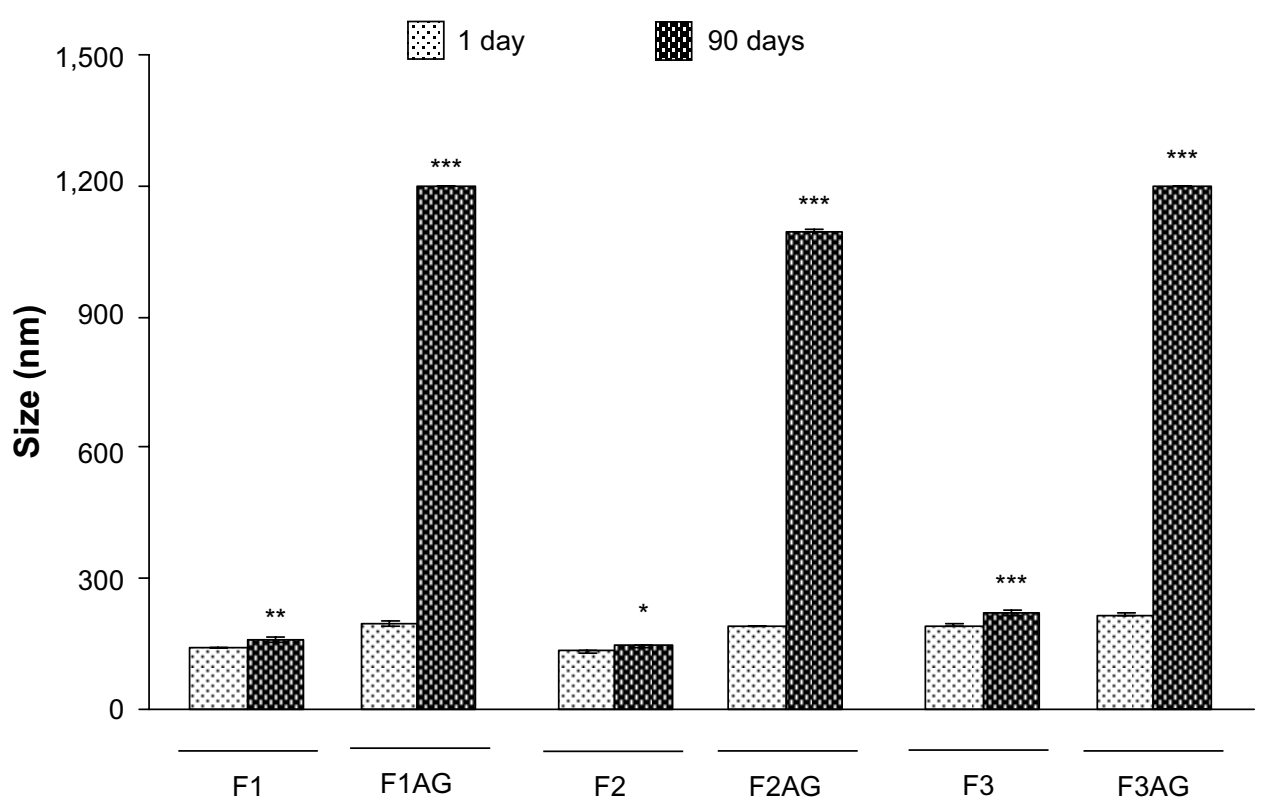

Figure 4 The physical stability of nonionic surfactant vesicles at $25^{\circ} \mathrm{C}$. Their average size and zeta potential were measured using dynamic light scattering at different time points (days) at $25^{\circ} \mathrm{C}$. The data are the average \pm standard error of the mean of three experiments.

Notes: $* P<0.05 ; * * P<0.01 ; * * * P<0.001$

Abbreviations: PDI, polydispersity index; AG, monoammonium glycyrrhizinate solution $(1 \% \mathrm{w} / \mathrm{v})$.

reducing edema and pain in appropriate animal models. AG shows anti-inflammatory activity in vivo, which is improved further when formulated in topical vesicular carriers. ${ }^{23,32,43}$ Our group has previously demonstrated that topical penetration of AG formulated as NSVs and/or ethosomes is affected by the anatomical structure of the skin. Furthermore, ethosomes and NSVs are not toxic and are well tolerated by human volunteers. ${ }^{23,32}$

In vitro and in vivo administration of NSVs can also be affected by interaction with cellular membranes and their internalization inside biological compartments. In fact, biological barriers and the cellular microenvironment can dramatically affect the biopharmaceutical features, metabolism, and therapeutic efficacy of NSVs. In this study, our group demonstrated that Tween 20 NSVs can be used to prepare stable and $\mathrm{pH}$-sensitive vesicles capable of interacting with biological membranes. ${ }^{34}$ Although we have previously demonstrated that Tween 20 and/or Tween 21 combined with cholesterol and CHEMS can yield $\mathrm{pH}-$ sensitive NSVs, ${ }^{8}$ in this study we evaluated the physicochemical properties of NSVs further to investigate the effect of an anti-inflammatory drug (AG) on vesicular architecture. Our results demonstrate that AG-loaded NSVs did not affect the zeta potential or bilayer microfluidity, whereas the average size and polydispersity index increased when AG was added into the lipid bilayer. 


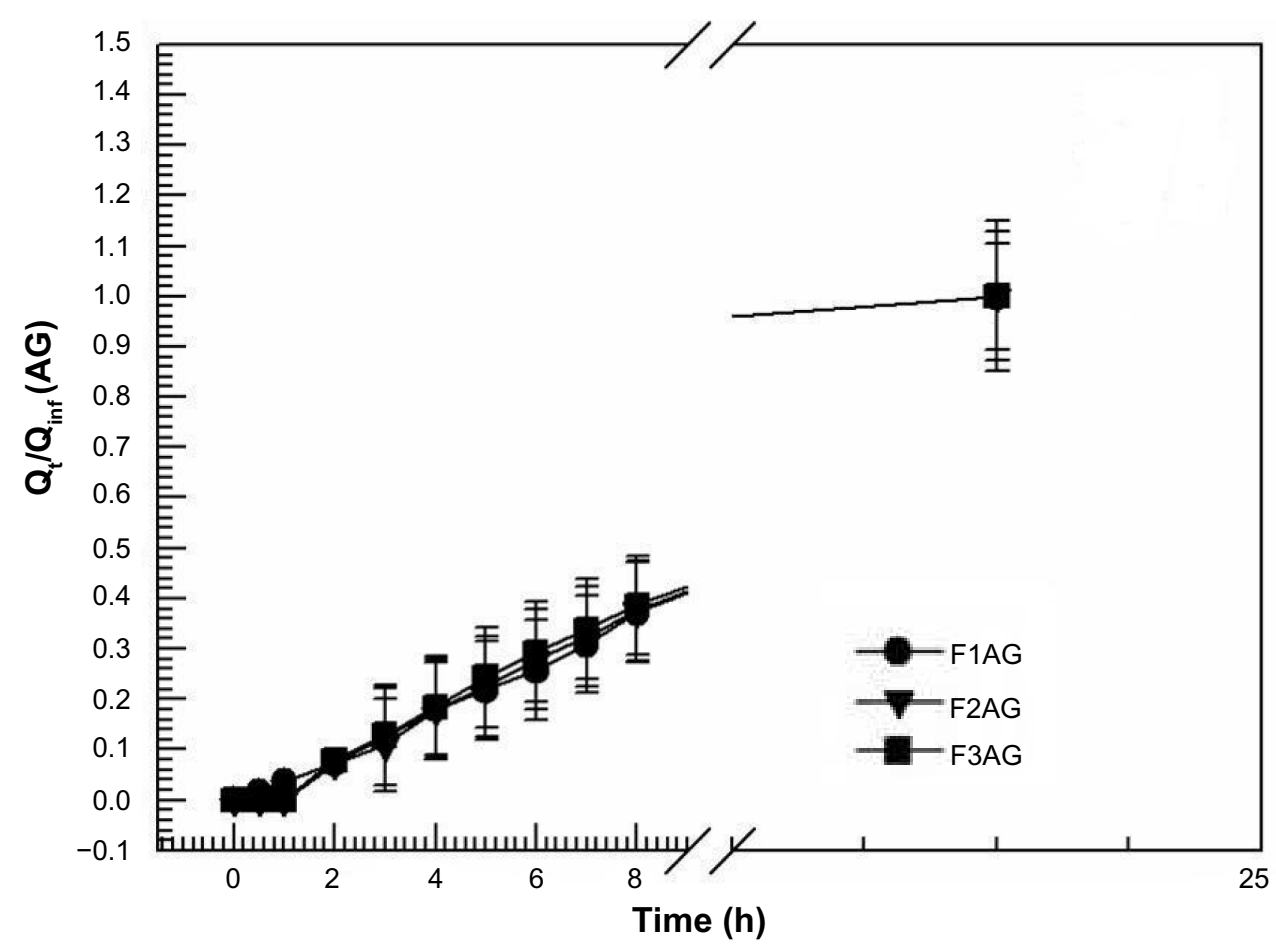

Figure 5 In vitro release of AG from nonionic surfactant vesicles. The release study was carried out at 24 hours (h) and $37^{\circ} \mathrm{C} \pm 0.5^{\circ} \mathrm{C}$. The release of $\mathrm{AG}$ from nonionic surfactant vesicles was evaluated measuring the amount of anti-inflammatory drug as a function of time. The release of AG from the nonionic surfactant vesicles was calculated as the amount of drug released over time with respect to the total amount of drug added into the formulation. The data are the average \pm standard error of the mean of three experiments.

Abbreviations: AG, monoammonium glycyrrhizinate solution ( $1 \% \mathrm{w} / \mathrm{v}) ; \mathrm{Q}_{\mathrm{t}}$, amount released at time $\mathrm{t}$; $\mathrm{Q}_{\text {inf }}$ amount released after vesicles disruption.

These findings are not consistent with previously reported data. ${ }^{23,43}$ Self-assembling of Span 20 and Tween 85 surfactants into NSVs and entrapping of AG achieved a decrease in average size and polydispersity index and a slight decrease in zeta potential. This effect may depend on the hydrophobic properties of Span 20 and Tween 85, which could partially prevent interaction between the surfactants and $\mathrm{AG}$ due to $\log \mathrm{P}$ values. Furthermore, the fluorescence anisotropy in Span 20 and Tween 85 niosomes was increased, and this improvement may depend on different drug partitioning inside the bilayer. ${ }^{31,43}$ Differences between the data reported in the literature and those for our formulations may
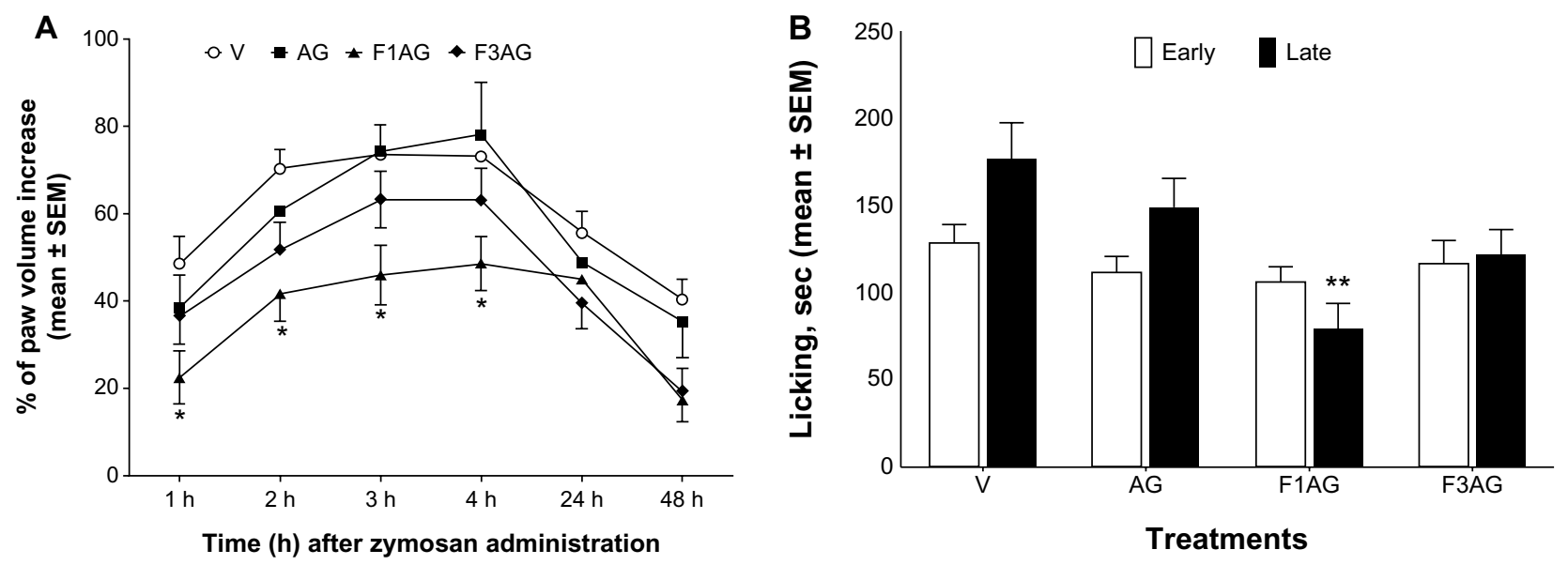

Figure 6 In vivo effects of AG-loaded nonionic surfactant vesicles on edema induced by zymosan (A) and on formalin-induced nociception (B). AG-loaded nonionic surfactant vesicles (FIAG and F3AG formulations) were selected for in vivo experiments. Free AG was used as reference compound. Two-way analysis of variance followed by Bonferroni's post hoc comparisons was used to evaluate statistical significance in edema experiments ( $n=10-12)$ while one-way analysis of variance followed by Dunnett's multiple comparison test was used to evaluate statistical significance in both the early and late phases of the formalin test ( $n=9-10)$. The data are considered to be statistically significant for $* P<0.05$ and $* * P<0.01$ versus animals treated with HEPES buffer $(\mathrm{V})$ or $\mathrm{FI}$ and $\mathrm{F} 3$ empty vesicles (data not shown).

Abbreviations: AG, monoammonium glycyrrhizinate solution (I\% w/v); SEM, standard error of the mean; h, hours; sec, seconds. 

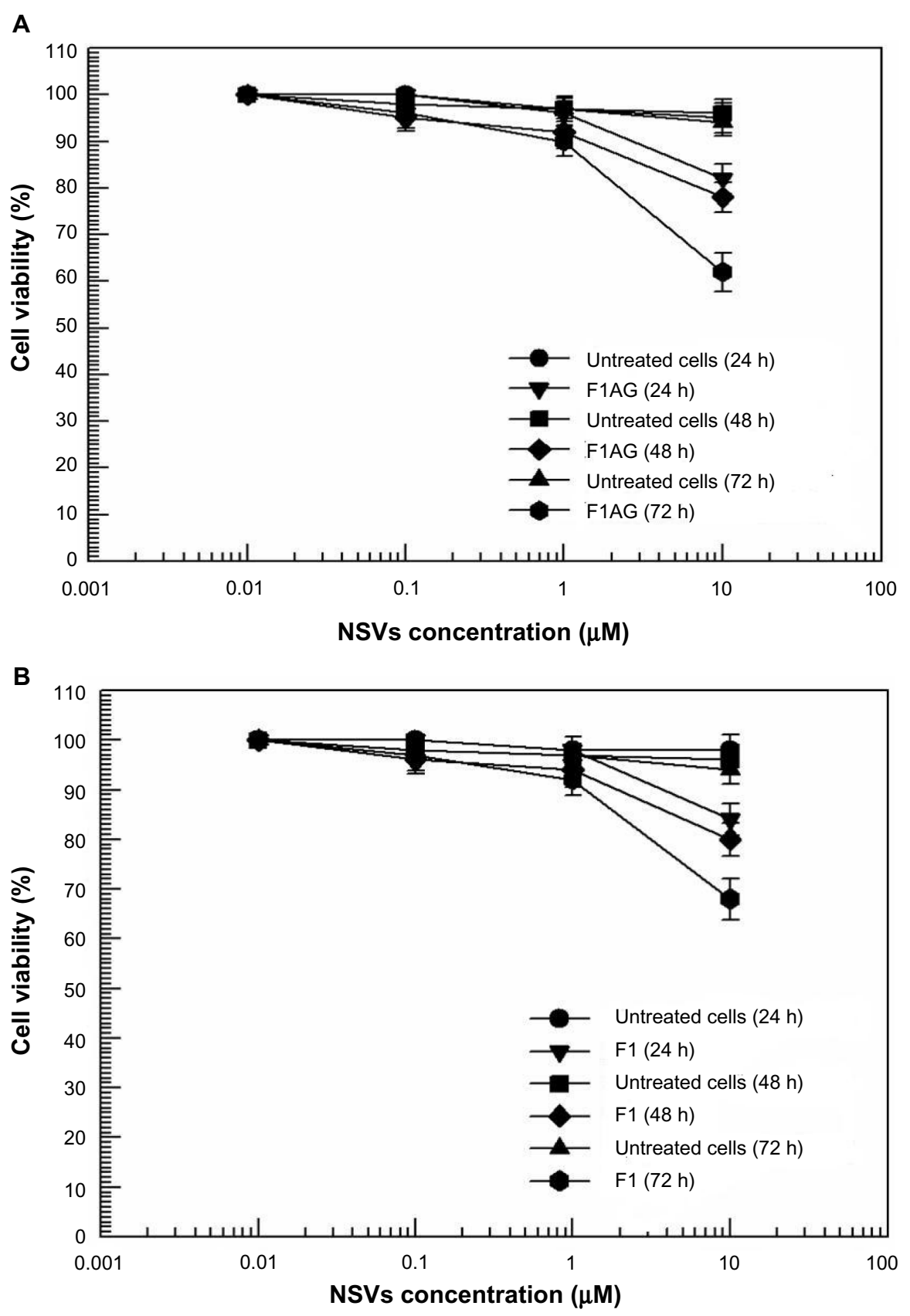

Figure 7 Cytotoxicity of primary human dermal fibroblasts. (A) In vitro tolerability of AG-loaded nonionic surfactant vesicles in primary human dermal fibroblasts. Cell viability was evaluated as a function of NSVs concentration $(0.0 \mathrm{I}, 0.1$, I, and I0 $\mu \mathrm{M})$ and time of incubation ( 24,48 and 72 hours). Primary human dermal fibroblasts were treated using FIAG ( $\% \mathrm{w} / \mathrm{v}$ at concentration). Safety and tolerability of nonionic surfactant vesicles were evaluated calculating the percentage of cellular viability with MTT assay. Untreated cells are used as the control. Error bars, if not shown, are within symbols. The data are the average of six experiments \pm the standard error of the mean.

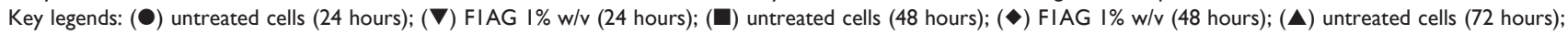
(1) FIAG I\% w/v (72 hours). (B) In vitro tolerability of empty nonionic surfactant vesicles on primary human dermal fibroblasts. The cell viability was evaluated as a function of nonionic surfactant vesicle concentration $(0.0 \mathrm{I}, 0 . \mathrm{I}$, I, and $10 \mu \mathrm{M})$ and time of incubation $(24,48$, and 72 hours). The primary human dermal fibroblasts were treated using NSVs. Safety and tolerability of nonionic surfactant vesicles were evaluated calculating the percentage of cellular viability with MTT assay. Control represents untreated cells. Error bars, if not shown, are within symbols. Data are the average of six experiments \pm SEM. Key legends: $(\mathbf{0})$ untreated cells (24 hours); ( $\mathbf{\nabla})$ FI (24 hours); ( $\square)$ untreated cells (48 hours); ( ) FI (48 hours); ( $\mathbf{\Delta}$ ) untreated cells (72 hours); ( $)$ FI (72 hours).

Abbreviations: NSVs, nonionic surfactant vesicles; SEM, standard error of the mean; h, hours; AG, monoammonium glycyrrhizinate solution (I\% w/v).

reflect the physicochemical properties of the surfactants self-assembled into the NSVs. In particular, AG was preferentially distributed into the aqueous core of the NSVs rather than into the vesicle bilayers, and a different amount of drug was adsorbed onto the vesicle surface, as demonstrated by the zeta potential values, potentially affecting entrapment efficiency. The NSVs remain stable for at least 90 days at $4^{\circ} \mathrm{C}$ but were not stable at $25^{\circ} \mathrm{C}$. In addition, AG-loaded NSVs do not modify the physicochemical features of Tween 20 NSVs in serum. 
As reported in the literature for other colloidal carriers, particle aggregation affects the physical stability of NSVs due to modification of the surface charge on NSVs. ${ }^{44,45}$ In particular, highly negative zeta potential values generally prevent aggregation and stabilize nanocarriers. Long-term physical stability of colloidal suspensions is obtained at a minimum zeta potential of $-30 \mathrm{mV}$, which allows for suitable electrostatic repulsion between monodispersed nanoparticles. ${ }^{46}$

Turbiscan Lab Expert was used to predict the longterm stability of the NSVs. $\triangle \mathrm{BS}$ profiles demonstrated no significant negative or positive variations in signals during scanning of the samples. In particular, $\Delta \mathrm{BS}$ could be overlapped with the baseline value and do not exceed $2 \%$ during analysis. This value was below $10 \%$, representing a threshold value for positive and negative signals and indicating a stable formulation. ${ }^{27,47,48}$ The NSVs showed an $\triangle \mathrm{BS}$ profile similar to that of NSVs loaded with beclomethasone dipropionate, another anti-inflammatory drug, and ultradeformable vesicles containing linoleic unsaturated fatty acid, as previously reported. ${ }^{29,49}$ Entrapment of AG (1\% w/v) does not affect the long-term stability of NSVs. In fact, AG-loaded NSVs showed a $\triangle B S$ profile similar to that obtained for the empty formulations. The results obtained using Turbiscan Lab Expert were in agreement with those from dynamic light scattering analysis, and allowed prediction of the longterm stability of NSVs without using traditional invasive procedures. ${ }^{50-52}$

In vitro AG release and the bilayer fluidity of NSVs do not depend on niosomal composition. Instead, the AG lag time may depend on compartmentalization of $A G$ inside the aqueous core of the NSVs as well as a slow drug permeation coefficient $\left(\mathrm{P}_{\mathrm{b}}\right)$ through the vesicle bilayer.

The release profile of AG was different from that previously reported for NSVs prepared using bola surfactants, whereby AG showed two-phase kinetics with rapid desorption of the drug from the NSV surface followed by a constant release rate. ${ }^{53} \mathrm{AG}$ was released from bola-surfactant NSVs within the first 3 hours, indicating zero-order release kinetics. The release profile of AG from bola-surfactant NSVs was also constant until 8 hours, indicating the kinetics of a colloidal reservoir system..$^{54-56}$

In vitro cell viability experiments using primary human dermal fibroblasts demonstrated that our NSVs did not have any significant cytotoxicity at varying incubation times (24, 48, and 72 hours) until a drug concentration of $1 \mu \mathrm{M}$ was reached. The lack of cytotoxicity seen for the empty vesicles may be attributable to the self-assembling of nonionic surfactants into colloidal structures, as has been reported previously. ${ }^{32,53}$ Cytotoxicity to primary human dermal fibroblasts occurred using the same concentration of nonionic surfactants $(1 \mu \mathrm{M})$ and by extending the incubation time over 24 hours. The data obtained demonstrate that cytotoxicity is time-dependent and is further increased at 72 hours of incubation ( $\sim 60 \%$ for empty NSVs and AG $1 \% \mathrm{w} / \mathrm{v}$ NSVs). The results also demonstrate that there is no significant difference in cytotoxicity between empty NSVs and AG-loaded NSVs in primary human dermal fibroblasts at 72 hours. AG does not affect per se cytotoxicity. ${ }^{57,58}$

In vivo experiments showed different activity for the F1AG and F3AG formulations. F1AG strengthens antinociceptive effects and further decreases edema in murine models compared with the animal group administered AG and empty NSVs. In contrast, treatment with F3AG did not have any statistically significant effect on antinociception or edema when compared with control and empty NSVs. In vivo differences between F1AG and F3AG do not depend on the physicochemical features of the NSVs and are affected by the anti-inflammatory activity of AG in experimental models in vivo. ${ }^{59,60}$ In fact, the anti-inflammatory effects of AG occurred within 3 hours; while pH-sensitive NSVs (F3AG) allowed the increase of AG inside the inflammatory area where the payload is rapidly released. The different release kinetics of F1AG and F3AG were found to be due to CHEMS, which can modify the NSV bilayer at acidic $\mathrm{pH}$, thus promoting rapid release of payloads. ${ }^{61} \mathrm{pH}$ modification occurring in an inflamed area can enable rapid release of AG from the F3 formulation, thus having anti-inflammatory and antinociceptive effects similar to those achieved using free AG. Conversely, neutral NSVs (F1AG) are not affected by $\mathrm{pH}$ modification and allow slow release of $\mathrm{AG}$ in the inflamed area, thus strengthening the antinociceptive effects and reducing edema in murine models. In addition, the experimental setup allowed comparison of our results with those reported previously ${ }^{32}$ and demonstrates that NSVs made from Tween 20 improved the in vivo anti-inflammatory activity and antinociceptive effects of AG, like Tween 85 and Span $20 .{ }^{32}$ In addition, the presence of CHEMS provides preliminary data for further investigation of the in vivo anti-inflammatory activity and antinociceptive effects of $\mathrm{pH}$-sensitive NSVs.

\section{Conclusion}

This study demonstrates that neutral or $\mathrm{pH}$-sensitive NSVs can be used as nanotherapeutics for anti-inflammatory and antinociceptive therapy. AG-loaded NSVs showed suitable in vitro and in vivo physicochemical features for therapeutic 
application and are stable in biological fluids. Our results show that AG-loaded NSVs containing Tween 20/cholesterol improve the anti-inflammatory activity and antinociceptive effects of AG in comparison with the free drug. AG-loaded NSVs are nontoxic to primary human dermal fibroblasts in vitro and in vivo in a murine animal model, and could also be used as a suitable nanotherapeutic for preclinical studies. Further experiments are being developed using appropriate animal models to investigate the anti-inflammatory activity and antinociceptive efficacy of $\mathrm{pH}$-sensitive NSVs in vivo.

\section{Acknowledgments}

This research was supported by a grant from the Interregional Research Center for Food Safety and Health (PONa3_00359). The authors are grateful to DR Giuseppe Pittari (Research Center for Food Safety and Health) for the statistical analysis.

\section{Disclosure}

The authors report no conflicts of interest in this work.

\section{References}

1. Uchegbu IF, Vyas SP. Non-ionic surfactant based vesicles (niosomes) in drug delivery. Int J Pharm. 1998;172(1-2):33-70.

2. Terzano C, Allegra L, Alhaique F, Marianecci C, Carafa M. Non phospholipid vesicles for pulmonary glucocorticoid delivery. Eur J Pharm Biopharm. 2005;59(1):57-62.

3. Di Marzio L, Esposito S, Rinaldi F, Marianecci C, Carafa M. Polysorbate 20 vesicles as oral delivery system: in vitro characterization. Colloids Surf B Biointerfaces. 2013;104:200-206.

4. Di Marzio L, Marianecci C, Rinaldi F, Esposito S, Carafa M. Deformable surfactant vesicles loading ammonium glycyrrhizinate: characterization and in vitro permeation studies. Lett Drug Des Discov. 2012;9(5):494-499.

5. Marianecci C, Rinaldi F, Di Marzio L, et al. Polysorbate 20 vesicles as multi-drug carriers: in vitro preliminary evaluations. Lett Drug Des Discov. 2013;10(3):212-218.

6. Mahale NB, Thakkar PD, Mali RG, Walunj DR, Chaudhari SR. Niosomes: novel sustained release nonionic stable vesicular systems an overview. Adv Colloid Interface Sci. 2012;183-184:46-54.

7. Marianecci $C$, Rinaldi $F$, Ingallina $C$, et al. Smart magnetic nanovesicles for theranostic application: preparation and characterization. Nuovo Cimento C. 2013;36C(2):103-110.

8. Di Marzio L, Marianecci C, Petrone M, Rinaldi F, Carafa M. Novel pHsensitive non-ionic surfactant vesicles: comparison between Tween 21 and Tween 20. Colloids Surf B Biointerfaces. 2011;82(1):18-24.

9. Carafa M, Di Marzio L, Marianecci C, et al. Designing novel pHsensitive non-phospholipid vesicle: characterization and cell interaction. Eur J Pharm Sci. 2006;28(5):385-393.

10. Baronzio G, Schwartz L, Kiselevsky M, et al. Tumor interstitial fluid as modulator of cancer inflammation, thrombosis, immunity and angiogenesis. Anticancer Res. 2012;32(2):405-414.

11. Wiig H. Pathophysiology of tissue fluid accumulation in inflammation. J Physiol. 2011;589 Pt 12:2945-2953.

12. Torchilin VP. Multifunctional nanocarriers. Adv Drug Deliv Rev. 2012;64 Suppl:302-315.

13. Lee ES, Gao Z, Bae YH. Recent progress in tumor $\mathrm{pH}$ targeting nanotechnology. J Control Release. 2008;132(3):164-170.
14. Huang S, Shao K, Kuang Y, et al. Tumor targeting and microenvironmentresponsive nanoparticles for gene delivery. Biomaterials. 2013;34(21):5294-5302.

15. Tannock IF, Rotin D. Acid pH in tumors and its potential for therapeutic exploitation. Cancer Res. 1989;49(16):4373-4384.

16. Roy CR, Salcedo SP, Gorvel JP. Pathogen-endoplasmic-reticulum interactions: in through the out door. Nat Rev Immunol. 2006;6(2): 136-147.

17. Luzio JP, Rous BA, Bright NA, Pryor PR, Mullock BM, Piper RC. Lysosome-endosome fusion and lysosome biogenesis. J Cell Sci. 2000;113 Pt 9:1515-1524.

18. Ciechanover A. Intracellular protein degradation: from a vague idea thru the lysosome and the ubiquitin-proteasome system and onto human diseases and drug targeting. Cell Death Differ. 2005;12(9): $1178-1190$.

19. Torchilin VP. Multifunctional and stimuli-sensitive pharmaceutical nanocarriers. Eur J Pharm Biopharm. 2009;71(3):431-444.

20. Masotti A, Vicennati P, Alisi A, et al. Novel Tween ${ }^{\circledR} 20$ derivatives enable the formation of efficient $\mathrm{pH}$-sensitive drug delivery vehicles for human hepatoblastoma. Bioorg Med Chem Lett. 2010;20(10): 3021-3025

21. Matsui S, Matsumoto H, Sonoda Y, et al. Glycyrrhizin and related compounds down-regulate production of inflammatory chemokines IL-8 and eotaxin 1 in a human lung fibroblast cell line. Int Immunopharmacol. 2004;4(13):1633-1644.

22. Genovese T, Menegazzi M, Mazzon E, et al. Glycyrrhizin reduces secondary inflammatory process after spinal cord compression injury in mice. Shock. 2009;31(4):367-337.

23. Paolino D, Lucania G, Mardente D, Alhaique F, Fresta M. Ethosomes for skin delivery of ammonium glycyrrhizinate: in vitro percutaneous permeation through human skin and in vivo anti-inflammatory activity on human volunteers. J Control Release. 2005;106(1-2):99-110.

24. Carafa M, Santucci E, Alhaique F, et al. Preparation and properties of new unilamellar non-ionic/ionic surfactant vesicles. Int J Pharm. 1998;160(1):51-59.

25. Di Marzio L, Marianecci C, Cinque B, et al. pH-sensitive non-phospholipid vesicle and macrophage-like cells: binding, uptake and endocytotic pathway. Biochem Biophys Acta. 2008;1778(12):2749-2756.

26. Carafa M, Santucci E, Lucania G. Lidocaine-loaded non-ionic surfactant vesicles: characterization and in vitro permeation studies. Int J Pharm. 2002;231(1):21-32.

27. Celia C, Trapasso E, Cosco D, Paolino D, Fresta M. Turbiscan Lab ${ }^{\circledR}$ Expert analysis of the stability of ethosomes ${ }^{\circledR}$ and ultradeformable liposomes containing a bilayer fluidizing agent. Colloids Surf B Biointerfaces. 2009;72(1):155-160.

28. Ho NFH, Ganesan MG, Weiner ND, et al. Mechanism of topical delivery of liposomally entrapped drugs. J Control Release. 1985;2(1):61-65.

29. Marianecci C, Paolino D, Celia C, et al. Non-ionic surfactant vesicles in pulmonary glucocorticoid delivery: characterization and interaction with human lung fibroblasts. J Control Release. 2010;147(1):127-135.

30. Papini S, Cecchetti D, Campani D, et al. Isolation and clonal analysis of human epidermal keratinocyte stem cells in long-term culture. Stem Cells. 2003;21(4):481-494.

31. Paolino D, Cosco D, Cilurzo F, et al. Improved in vitro and in vivo collagen biosynthesis by asiaticoside-loaded ultradeformable vesicles. J Control Release. 2012;162(1):143-151.

32. Marianecci C, Rinaldi F, Mastriota M, et al. Anti-inflammatory activity of novel ammonium glycyrrhizinate/niosomes delivery system: human and murine models. J Control Release. 2012;164(1):17-25.

33. Colucci M, Maione F, Bonito MC, Piscopo A, Di Giannuario A, Pieretti S. New insights of dimethyl sulphoxide effects (DMSO) on experimental in vivo models of nociception and inflammation. Pharmacol Res. 2008;57(6):419-425.

34. Pieretti S, Di Giannuario A, De Felice M, Perretti M, Cirino G. Stimulusdependent specificity for annexin 1 inhibition of the inflammatory nociceptive response: the involvement of the receptor for formylated peptides. Pain. 2004;109(1):52-63. 
35. Marianecci C, Rinaldi F, Di Marzio L, et al. Interaction of $\mathrm{pH}$-sensitive non-phospholipid liposomes with cellular mimetic membranes. Biomed Microdevices. 2013;15(2):299-309.

36. Wang M, Yuan Y, Gao Y, et al. Preparation and characterization of 5-fluorouracil $\mathrm{pH}$-sensitive niosome and its tumor-targeted evaluation: in vitro and in vivo. Drug Dev Ind Pharm. 2012;38(9):1134-1141.

37. Carafa M, Marianecci C, Rinaldi F, Santucci E, Tampucci S, Monti D. Span and Tween neutral and $\mathrm{pH}$-sensitive vesicles: characterization and in vitro skin permeation. J Liposome Res. 2009;19(4):332-340.

38. Sinico C, Fadda AM. Vesicular carriers for dermal drug delivery. Expert Opin Drug Deliv. 2009;6(8):813-825.

39. Manconi M, Sinico C, Valenti D, Loy G, Fadda AM. Niosomes as carriers for tretinoin. I. Preparation and properties. Int J Pharm. 2002;234(1-2):237-248.

40. [No authors listed]. CFR Parts 182 and 184 GRAS status of licorice, (Glycyrrhiza), ammoniated glycyrrhizin and monoammonium glycyrrhizinate. Fed Regist. 1985;50:21043-21044.

41. Commission Decision of February 23, 1999 adopting a register of flavouring substances used in or on foodstuffs drawn up in application of Regulation (EC) No 2232/96 of the European Parliament and of the Council of October 28, 1996 (notified under number C(1999) 399) (text with EEA relevance) (1999/217/EC). Official Journal of the European Communities. 1999;L084:1-74. Available from: http://eur-lex.europa.eu/ LexUriServ/LexUriServ.do?uri=OJ:L:1999:084:0001:0137:EN:PDF. Accessed January 6, 2014.

42. Cosmetic Ingredient Review Expert Panel. Final report on the safety assessment of glycyrrhetinic acid, potassium glycyrrhetinate, disodium succinoyl glycyrrhetinate, glyceryl glycyrrhetinate, glycyrrhetinyl stearate, stearyl glycyrrhetinate, glycyrrhizic acid, ammonium glycyrrhizate, dipotassium glycyrrhizate, disodium glycyrrhizate, trisodium glycyrrhizate, methyl glycyrrhizate, and potassium glycyrrhizinate. Int J Toxicol. 2007;26 Suppl 2:79-112.

43. Wu Y, Yang W, Wang C, Hu J, Fu S. Chitosan nanoparticles as a novel delivery system for ammonium glycyrrhizinate. Int J Pharm. 2005;295(1-2):235-245.

44. Casals E, Galán AM, Escolar G, Gallardo M, Estelrich J. Physical stability of liposomes bearing hemostatic activity. Chem Phys Lipids. 2003;125(2):139-146.

45. Karmali PP, Simberg D. Interactions of nanoparticles with plasma proteins: implication on clearance and toxicity of drug delivery systems. Expert Opin Drug Deliv. 2011;8(3):343-357.

46. Muller RH, Jacobs C, Kayser O. Nanosuspensions as particulate drug formulations in therapy: rationale for development and what we can expect for the future. Adv Drug Deliv Rev. 2001;47(1):3-19.

47. Lemarchand C, Couvreur P, Vauthier C, Costantini D, Gref R. Study of emulsion stabilization by graft copolymers using optical analyzer Turbiscan. Int J Pharm. 2003;254(1):77-82.

48. Daoud-Mahammed S, Couvreur P, Gref R. Novel self-assembling nanogels: stability and lyophilisation studies. Int J Pharm. 2007; 332(1-2):185-191.
49. Celia C, Cilurzo F, Trapasso E, Cosco D, Fresta M, Paolino D Ethosomes $^{\circledR}$ and transfersomes ${ }^{\circledR}$ containing linoleic acid: physicochemical and technological features of topical drug delivery carriers for the potential treatment of melasma disorders. Biomed Microdevices. 2012;14(1):119-130.

50. Mengual O, Meunier G, Cayré I, Puech K, Snabre P. Characterization of instability of concentrated dispersions by new optical analyzer: the TURBISCAN MA 1000. Colloids and Surfaces A. 1999;152(1-2):111-123.

51. Mengual O, Meunier G, Cayré I, Puech K, Snabre P. TURBISCAN MA 2000: multiple light scattering measurement for concentrated emulsion and suspension instability analysis. Talanta. 1999;50(20):445-456.

52. Buron H, Mengual O, Meunier G, et al. Optical characterization of concentrated dispersions: applications and laboratory analysis and on line process to monitoring and control. Polym Int. 2004;53(9): 1205-1209.

53. Paolino D, Muzzalupo R, Ricciardi A, et al. In vitro and in vivo evaluation of Bola-surfactant containing niosomes for transdermal delivery. Biomed Microdevices. 2007;9(4):421-433.

54. Fattal E, Rojas J, Roblot-Treupel L, et al. Ampicillin-loaded liposomes and nanoparticles: comparison of drug loading, drug release and in vitro antimicrobial activity. J Microencapsul. 1991;8(1):29-36.

55. Araújo J, Garcia mL, Mallandrich M, et al. Release profile and transscleral permeation of triamcinolone acetonide loaded nanostructured lipid carriers (TA-NLC): in vitro and ex vivo studies. Nanomedicine. 2012;8(6):1034-1041

56. Xu Y, Jia Y, Wang Z, et al. Mathematical modelling and finite element simulation of slow release of drugs using hydrogels as carriers with various drug concentration distributions. J Pharm Sci. 2013;102(5): $1532-1543$.

57. Fiume Z. Final report on the safety assessment of lecithin and hydrogenated lecithin. Int J Toxicol. 2001;20 Suppl 1:21-45.

58. Szolcsányi J, Pintér E, Helyes Z, et al. Inhibition of the function of TRPV1-expressing nociceptive sensory neurons by somatostatin 4 receptor agonism: mechanism and therapeutical implications. Curr Top Med Chem. 2011;11(17):2253-2263.

59. Schlosburg JE, Kinsey SG, Lichtman AH. Targeting fatty acid amide hydrolase (FAAH) to treat pain and inflammation. AAPS $J$. 2009;11(1):39-44.

60. Lanigan RS, Yamarik TA, Cosmetic Ingredients Review Expert Panel. Final report on the safety assessment of PEG-6, 8, and 20 sorbitan beeswax. Int J Toxicol. 2001;20 Suppl 4:27-38.

61. Carmo VA, Ferrari CS, Reis EC, et al. Biodistribution study and identification of inflammation sites using $99 \mathrm{mTc}$-labelled stealth $\mathrm{pH}$-sensitive liposomes. Nucl Med Commun. 2008;29(1):33-38. 


\section{Supplementary materials}

Ammonium glycyrrhizinate (AG)-loaded niosomes as a potential nanotherapeutic system with anti-inflammatory activity in murine models.

\section{Physicochemical stability of NSVs measured by Turbiscan Lab ${ }^{\circledR}$ Expert}

The long-term stability of nonionic surfactant vesicles (NSVs) was evaluated using Turbiscan Lab Expert (Formulaction, L'Union, France) as previously reported. ${ }^{1}$ Briefly, $10 \mathrm{~mL}$ samples were loaded into glass cylinder tubes and analyzed using a pulsed near infrared light-emitting diode source at a wavelength of $880 \mathrm{~nm}$. Two synchronous optical detectors transmitting and back scattering incident light at $180^{\circ}$ and $45^{\circ}$, respectively, were used during the analysis. Samples were scanned until $10 \mathrm{~mm}$ in height and detection was performed every minute for one hour. The analysis was performed as a function of back scattering (BS) of NSVs (Supplementary material). BS was evaluated using the following equation:

$$
\mathrm{BS}=\frac{1}{\sqrt{\lambda^{*}}}
$$

where $\lambda^{*}$ was the photon transport mean free path in the sample dispersion. Additionally, $\lambda^{*}(\Phi, \mathrm{d})$ was determined based on the following equation:

$$
\lambda *(\phi, d)=\frac{2 d}{3 \phi(1-g) Q_{S}}
$$

where $\Phi$ and $\mathrm{d}$ were the volume fraction and diameter $(\mathrm{nm})$ of particles, respectively; $\mathrm{g}, \mathrm{d}$, and $\mathrm{Q}_{\mathrm{S}}$ were optical parameters extrapolated by Mie theory. BS was finally reported as $\triangle \mathrm{BS}$ and the data were exported using Turbiscan EasySoft Converter.

\section{Physicochemical and serum stability of NSVs}

Physicochemical stability studies of AG-loaded NSVs were carried out by evaluating the average size, polydispersity index, and zeta potential of NSVs stored at $4^{\circ} \mathrm{C}$ and $25^{\circ} \mathrm{C}$ for 90 days. Samples were analyzed at different time points $(1,30,60$, and 90 days) and average size and zeta potential were measured as previously reported. ${ }^{2}$

Serum stability was further evaluated by coincubating AG-loaded NSVs $(250 \mu \mathrm{L})$ diluted in $2.25 \mathrm{~mL}$ of HEPES buffer $(10 \mathrm{mM}, \mathrm{pH} 7.4)$ supplemented with $(10 \% \mathrm{v} / \mathrm{v})$ and without $(0 \% \mathrm{v} / \mathrm{v})$ fetal bovine serum. The percentage of $A G$ leaked from the NSVs was measured 3 and 24 hours after incubation by dissolving NSVs with isopropyl alcohol.

The concentration of AG loaded into the NSVs was measured using high-performance liquid chromatography as previously reported. ${ }^{3} \mathrm{~A}$ liquid chromatographic system (Model 251 Hewlett-Packard, Milan, Italy) equipped with a $20 \mu \mathrm{L}$ Rheodyne model 7125 injection valve (Rheodyne, Rohnert Park, CA, USA) was used. The analysis was performed at room temperature using a reverse phase C-18 column $(3 \mu \mathrm{m}, 150 \times 4.6 \mathrm{~mm}$ id, Supelco Inc, St Louis, MO, USA). The high-performance liquid chromatography column was connected to an ultraviolet-visible detector set at a $254 \mathrm{~nm}$ wavelength ( $\lambda_{\max }$ of AG). The mobile phase consisted of acetonitrile/water/0.1 N phosphoric acid $(79.95 / 20 / 0.05 \mathrm{v} / \mathrm{v} / \mathrm{v})$ and was performed at a flow rate of $0.5 \mathrm{~mL}$ per minute. The AG showed a retention time of 3.97 minutes. The AG concentration was calculated using the following calibration curve:

$$
y=3107 x+47692
$$

where $\mathrm{y}$ is the peak area and $\mathrm{x}$ is the drug concentration $(\mathrm{mM})$, with $r^{2}=0.9999$.

The limits of detection and quantitation of AG were estimated by injecting samples at different concentrations $(0.2-100 \mu \mathrm{g} / \mathrm{mL})$, and were 0.13 and 0.3 , respectively. The accuracy was $99.76 \%$ with a specificity of 1.3 . The average size, polydispersity index, and zeta potential of the AG-loaded NSVs were further evaluated after incubation in serum.

\section{Drug entrapment efficiency of NSVs and in vitro release studies}

AG-loaded NSVs were evaluated using high-performance liquid chromatography as previously reported. ${ }^{4}$ Purified NSVs were disrupted using isopropyl alcohol (NSVs/isopropyl alcohol 1:1 v/v) and entrapment efficiency was quantified using the following equation:

$$
\text { Entrapment efficiency }=\frac{\operatorname{Drug}_{\text {Ent }}}{\operatorname{Drug}_{\text {Tot }}} \times 100
$$

where Drug Ent $_{\text {is }}$ the amount of AG (mg) entrapped inside NSVs and Drug Tot $_{\text {is }}$ the amount of AG (mg) added during the preparation procedure. Empty NSVs were used as a blank during the high-performance liquid chromatography experiments. The samples were analyzed and processed as herein reported.

In vitro release experiments were carried out using a flow-through apparatus (USP 35 th ed) at $37^{\circ} \mathrm{C}$ with HEPES 
buffer (10 mM, pH 7.4) as the receptor solution. One milliliter of NSVs was loaded inside cellulose membrane dialysis tubing (cutoff molecular weight 8,000) with a $5.5 \mathrm{~cm}^{2}$ diffusing area. Free AG was used as a control to confirm that the dialysis membrane did not interfere with diffusion. The AG concentration in the receptor medium was measured using high-performance liquid chromatography at different time points (1-10 hours). The release profile of AG was evaluated according to the following equation and monitored over time:

$$
\mathrm{Q}_{\mathrm{n}}=\mathrm{C}_{\mathrm{n}} \mathrm{V} \sum_{\mathrm{i}=1}^{\mathrm{n}-1} \mathrm{~V}_{\mathrm{s}} \mathrm{C}_{\mathrm{i}}
$$

where $\mathrm{C}_{\mathrm{i}}$ and $\mathrm{C}_{\mathrm{n}}$ are the $\mathrm{AG}$ concentrations measured from 1 to $\mathrm{n}, \mathrm{V}$ is the solution volume in the receptor compartment, and $\mathrm{V}_{\mathrm{s}}$ is the sample volume.

The permeability coefficient of the niosomal bilayer $\left(\mathrm{P}_{\mathrm{bl}}\right)$ was evaluated using a linear expression developed by Ho et $\mathrm{al}^{5}$ as previously reported:

$$
\mathrm{C}_{\mathrm{b}}=\mathrm{C}_{\mathrm{b}(0)}+\left(3 \mathrm{P}_{\mathrm{bl}} \mathrm{T} / \mathrm{a}(1-\varepsilon) \mathrm{V}\right) \mathrm{t}
$$

where, $\mathrm{C}_{\mathrm{b}}$ and $\mathrm{C}_{\mathrm{b}(0)}$ are the solute concentrations in the bulk liquid at time $t$ and $\mathrm{t}_{0}$, respectively; $\mathrm{T}$ is the initial total amount (mg) of AG-loaded NSVs; $\varepsilon$ is the volume fraction of the NSV pellet compared with the volume of NSV suspension measured by centrifugation at $22,000 \times g$ for 20 minutes at $4^{\circ} \mathrm{C}$, (J21-B centrifuge, Beckman Coulter Inc, Brea, CA, USA); $\mathrm{V}$ is the volume ( $\mathrm{mL}$ ) of NSV suspension and a is the NSV radius $(\mu \mathrm{m}) .{ }^{5}$ The equation developed by Ho et al can be applied for time points where a small amount of drug is released by vesicles. ${ }^{5}$

\section{In vitro cytotoxicity experiments}

\section{Primary human dermal fibroblast cell culture}

Primary human dermal fibroblasts were isolated from consenting patients who had undergone abdominal skin reduction surgery. ${ }^{6}$ Briefly, skin slides $(0.5 \times 0.5 \mathrm{~cm})$ pretreated with type IA collagenase $(1 \mathrm{mg} / \mathrm{mL})$ and type IVS hyaluronidase $(0.4 \mathrm{mg} / \mathrm{mL})$ at $37^{\circ} \mathrm{C}$ for 45 minutes were washed (in phosphate-buffered saline, $\mathrm{pH} 7.4$ ), seeded into $35 \mathrm{~mm}$ diameter tissue culture plates, and incubated for 14 hours using high-glucose Dulbecco's Modified Eagle's Medium (6 mL) supplemented with $(20 \% \mathrm{v} / \mathrm{v})$ fetal bovine serum, gentamicin, streptomycin, and penicillin. ${ }^{7}$ Cell culture medium $(10 \mathrm{~mL})$ was replaced every 3 days, allowing migration of primary human dermal fibroblasts from skin slides to the bottom of the cell culture plate. The cell culture medium and skin slides were removed and the primary human dermal fibroblasts were then incubated with fresh Dulbecco's Modified Eagle's Medium supplemented with fetal bovine serum $(10 \% \mathrm{v} / \mathrm{v})$, glutamine $(2 \mathrm{mmol} / \mathrm{L})$, penicillin $(50 \mathrm{U} / \mathrm{mL})$, and streptomycin $(50 \mu \mathrm{g} / \mathrm{mL})$ at $37^{\circ} \mathrm{C}$ in $5 \% \mathrm{CO}_{2}$. The medium was replaced every 2 days until $\sim 70 \%$ confluence was reached. Cellular morphology and the presence of microbial contaminants was checked during incubation using a microscope. Primary human dermal fibroblasts $\left(8 \times 10^{3}\right.$ cells $\left./ \mathrm{cm}^{2}\right)$ were seeded into 12 -well plastic culture dishes before the in vitro experiments.

\section{Cytotoxicity of NSVs}

The cytotoxicity of empty NSVs in primary human dermal fibroblasts was evaluated using Trypan blue dye exclusion (cell mortality) and MTT (cell viability) assays. Primary human dermal fibroblasts were seeded into 12-well plastic culture dishes $\left(8 \times 10^{3}\right.$ cells $\left./ \mathrm{cm}^{2}\right)$ for the Trypan blue dye exclusion assay and 96-well tissue culture dishes $\left(5.6 \times 10^{3}\right.$ cells $\left./ \mathrm{cm}^{2}\right)$ for the MTT assay. After 24 hours of incubation, the cell culture medium was replaced with fresh medium and empty NSVs were added at different concentrations $(0.01,0.1,1,10 \mu \mathrm{M})$. NSVs were calculated as a function of Tween 20 self-assembling into NSVs. Cytotoxicity experiments were carried out at different time points (ie, 24, 48, and 72 hours). In the Trypan blue dye exclusion assay, primary human dermal fibroblasts were harvested using trypsin/ethylenediamine tetraacetic acid solution and washed twice with phosphate-buffered saline ( $\mathrm{pH} 7.4)$. Eight milliliters of cellular suspension treated with empty NSVs were collected. Samples were then centrifuged using a Megafuge 1.0 (Heraeus Sepatech, DJB Labcare Ltd, Newport Pagnell, UK) at $800 \times g$ for 10 minutes at $4^{\circ} \mathrm{C}$. The supernatant was withdrawn and the pellet was suspended in $200 \mu \mathrm{L}$ of Trypan blue buffer. The amount of dead cells (blue-stained) was counted using a hemocytometer chamber and an optical microscope (Labophot-2, Nikon, Tokyo, Japan). Percentage cell death was calculated using the following equation:

$$
\text { Cell death }(\%)=\frac{D_{c}}{T_{c}} \times 100
$$

where $D_{c}$ is the number of dead cells and $T_{c}$ is the total number of cells.

Cell viability was performed using the MTT assay. Primary human dermal fibroblasts were seeded into 96-well culture dishes $\left(5.6 \times 10^{3}\right.$ cells $\left./ \mathrm{cm}^{2}\right)$ for 24 hours at $37^{\circ} \mathrm{C}$ in $5 \% \mathrm{CO}_{2}$. After 24 hours of incubation, the cell culture medium was replaced with fresh medium or empty NSVs; 
A

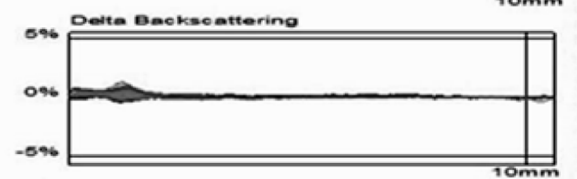

C
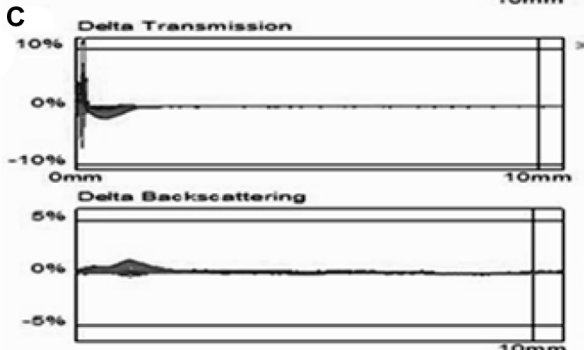

E
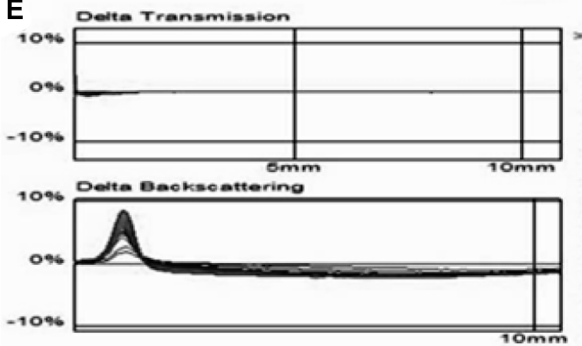

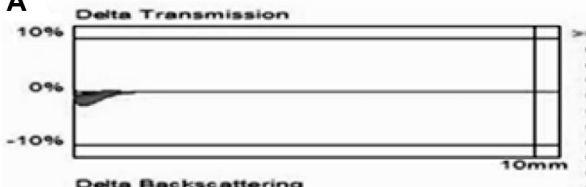

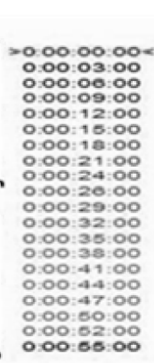

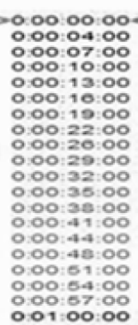

:00:57:00

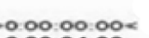

0000

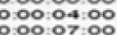

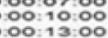
. 인 $00: 29: 00$ :00:32:00 00000

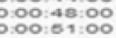
0:01:67:00

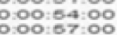

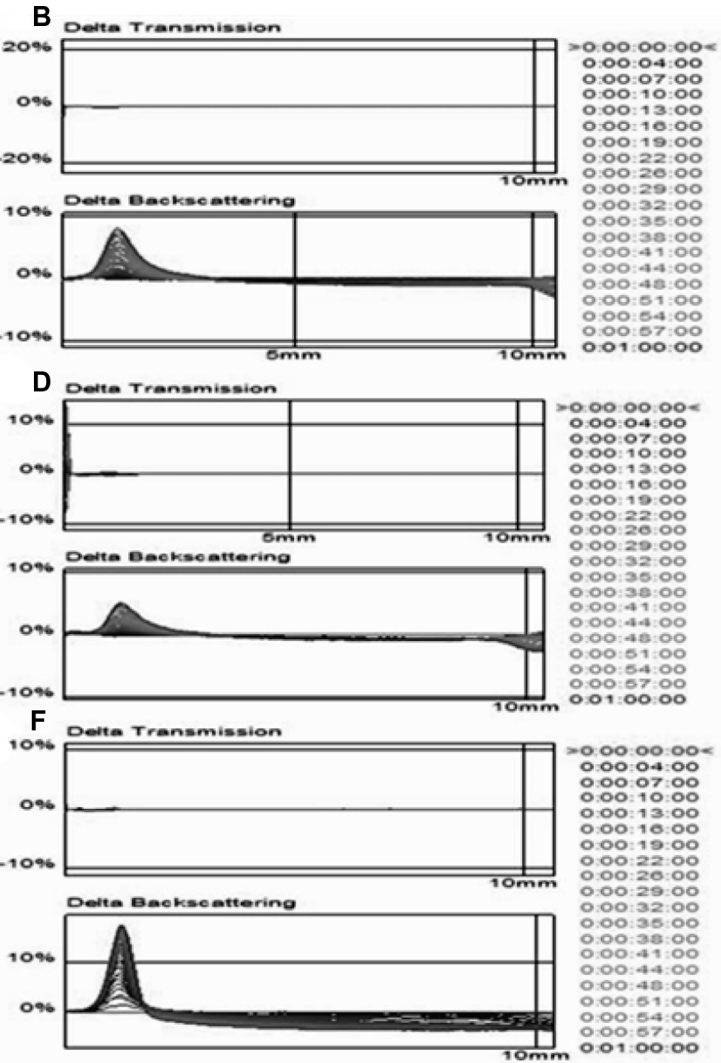

Figure SI Panel Turbiscan. Transmission and back scattering profile of formulation I (A), formulation I loading AG (B), formulation 2 (C), formulation 2 loading AG (D), formulation $3(\mathbf{E})$, and formulation 3 loading AG $(\mathbf{F})$ using Turbiscan Lab ${ }^{\circledR}$ Expert. Abbreviation: AG, monoammonium glycyrrhizinate solution (I\% w/v).

next, $10 \mu \mathrm{L}$ of MTT tetrazolium salt $(5 \mathrm{mg} / \mathrm{mL}$ in phosphatebuffered saline) were added to each well $(100 \mu \mathrm{L}$ per well) and further incubated for 3 hours, thus forming violet formazan crystals. Dimethylsulfoxide/ethanol $(1: 1 \mathrm{v} / \mathrm{v})(200 \mu \mathrm{L})$ was used to dissolve the obtained formazan crystals and the 96-well culture dishes were gently shaken at $230 \mathrm{rpm}$ (KS 130 Control, IKA ${ }^{\circledR}$ Werke GMBH and Co, Staufen, Germany) for 20 minutes. Cell viability was measured using an enzyme-linked immunosorbent assay microplate reader (Labsystem mod. Multiskan MS, Midland, ON, Canada) at an OD of $570 \mathrm{~nm}$. The percentage of viable cells was evaluated using the following equation:

$$
\% \text { cell viability }=\frac{\mathrm{Abs}_{\mathrm{T}}}{\mathrm{Abs}_{\mathrm{U}}} \times 100
$$

where $\mathrm{Abs}_{\mathrm{T}}$ is the absorbance of treated cells and $\mathrm{Abs}_{\mathrm{U}}$ is the absorbance of untreated (control) cells. Empty NSVs using the same Tween 20 concentration and untreated cells were used as the blank and control, respectively, during these experiments.
Table SI Serum stability expressed as AG release in the presence (I0\%) and absence of fetal bovine serum

\begin{tabular}{lll}
\hline Samples & \multicolumn{2}{c}{ AG release $\left(\mathbf{Q}_{\mathbf{t}} / \mathbf{Q}_{\text {inf }}\right)$ after $\mathbf{8}$ hours in } \\
\cline { 2 - 3 } & 0\% FBS & I0\% FBS \\
\hline FIAG & 0.38 & 0.38 \\
F2AG & 0.37 & 0.37 \\
F3AG & 0.39 & 0.39 \\
\hline
\end{tabular}

Abbreviations: AG, monoammonium glycyrrhizinate solution (I\% w/v); FBS, fetal bovine serum; $Q_{t}$, amount released at time $t ; Q_{\text {inf }}$ amount released after vesicle disruption.

\section{References} analysis of the stability of ethosomes ${ }^{\circledR}$ and ultradeformable liposomes containing a bilayer fluidizing agent. Colloids Surf B Biointerfaces. 2009;72(1):155-160

2. Caracciolo G, Pozzi D, Capriotti AL, et al. Factors determining the superior performance of lipid/DNA/protammine nanoparticles over lipoplexes. J Med Chem. 2011;54(12):4160-4171.

3. Paolino D, Lucania G, Mardente D, Alhaique F, Fresta M. Ethosomes for skin delivery of ammonium glycyrrhizinate: in vitro percutaneous permeation through human skin and in vivo anti-inflammatory activity on human volunteers. $J$ Control Release. 2005;106(1-2): 99-110.
1. Celia C, Trapasso E, Cosco D, Paolino D, Fresta M. Turbiscan Lab ${ }^{\circledR}$ Expert 
4. Marianecci C, Rinaldi F, Mastriota M, et al. Anti-inflammatory activity of novel ammonium glycyrrhizinate/niosomes delivery system: human and murine models. $J$ Control Release. 2012;164(1):17-25.

5. Ho NFH, Ganesan MG, Weiner ND, et al. Mechanism of topical delivery of liposomally entrapped drugs. J Control Release. 1985;2(1): $61-65$.
6. Marianecci C, Paolino D, Celia C, et al. Non-ionic surfactant vesicles in pulmonary glucocorticoid delivery: characterization and interaction with human lung fibroblasts. $J$ Control Release. 2010;147(1):127-135.

7. Papini S, Cecchetti D, Campani D, et al. Isolation and clonal analysis of human epidermal keratinocyte stem cells in long-term culture. Stem Cells. 2003;21(4):481-494.

\section{Publish your work in this journal}

The International Journal of Nanomedicine is an international, peerreviewed journal focusing on the application of nanotechnology in diagnostics, therapeutics, and drug delivery systems throughout the biomedical field. This journal is indexed on PubMed Central, MedLine, CAS, SciSearch ${ }^{\circledR}$, Current Contents ${ }^{\circledR} /$ Clinical Medicine,
Journal Citation Reports/Science Edition, EMBase, Scopus and the Elsevier Bibliographic databases. The manuscript management system is completely online and includes a very quick and fair peer-review system, which is all easy to use. Visit http://www.dovepress.com/ testimonials.php to read real quotes from published authors.

Submit your manuscript here: http://www.dovepress.com/international-journal-of-nanomedicine-journal 